\title{
Balancing conflicting requirements for grid and particle decomposition in continuum-Lagrangian solvers
}

\author{
Hariswaran Sitaraman ${ }^{\mathrm{a}, *}$, Ray Grout ${ }^{\mathrm{a}}$ \\ ${ }^{a}$ Computational Sciences Center, National Renewable Energy Laboratory, 15013 Denver \\ West Pkwy, Golden, CO, 80401
}

\begin{abstract}
Load balancing strategies for hybrid solvers that involve grid based partial differential equation solution coupled with particle tracking are presented in this paper. A typical Message Passing Interface (MPI) based parallelization of grid based solves are done using a spatial domain decomposition while particle tracking is primarily done using either of the two techniques. One of the techniques is to distribute the particles to MPI ranks to whose grid they belong to while the other is to share the particles equally among all ranks, irrespective of their spatial location. The former technique provides spatial locality for field interpolation but cannot assure load balance in terms of number of particles, which is achieved by the latter. The two techniques are compared for a case of particle tracking in a homogeneous isotropic turbulence box as well as a turbulent jet case. A strong scaling study is performed to more than 32,000 cores, which results in particle densities representative of anticipated exascale machines. The use of alternative implementations of MPI collectives and efficient load equalization strategies are studied to
\end{abstract}

\footnotetext{
${ }^{*}$ Corresponding author

Email address: Hariswaran.Sitaraman@nrel.gov (Hariswaran Sitaraman)
} 
reduce data communication overheads.

Keywords: Load balancing, Lagrangian particle tracking, Particle in Cell, Exascale simulations

\section{Introduction}

Hybrid continuum-Lagrangian solvers are used in turbulent reacting and multiphase flow simulations $[1,2,3,4]$, particle in cell (PIC) methods in

plasma simulations $[5,6,7,8]$ and in astrophysical simulations $[9,10]$. These involve calculation of grid based field variables through partial differential equation (PDE) solves and the movement of a large number of particles that represent droplets, charged particles or atoms through the domain using interpolated values of the velocity fields from the underlying computational grid. The update of seed particles using an interpolated vector field (typically velocity) defined on a grid is also used in the computation of stream lines as a post processing step in visualization [11].

The parallel decomposition of the grid based calculations is often the central concern and done primarily via spatial decomposition. This then constrains the particle decomposition to be a compromise, where the common approaches are limits of the locality/load balance trade off. One of the approaches for work sharing of particle calculations is by the spatial partitioning used for the PDE solve. MPI ranks own each of the grid partitions as well as the particles that are present within. Particles are transferred to neighboring ranks when they switch grids across partition boundaries. In many cases, the particle counts are similar to the number of grid points $[12,13]$. Hence, this method is well suited for the case of uniformly dis- 
tributed particles in space where the number of particles per grid partition is more or less equal. This situation is rarely seen in particle simulations, but does arise in gyrokinetic simulations of fusion plasmas [14] and early on in some cosmological calculations before particles start clustering in localized regions [15]. The co-locality of field data and particle positions for a given rank makes the process of exchanging data between the grid and particles efficient, because the data need not be transferred between ranks. For the case when the particles are clustered in regions of space, which occurs frequently in plasma PIC, turbulent reacting flow and astrophysical simulations, the spatial particle partitioning technique can be highly load imbalanced. An alternative technique is to partition all of the particles equally among all MPI ranks irrespective of their spatial locations. This achieves load balance in terms of particle movement calculations and field variable interpolation but spatial locality with respect to the grid is lost. Additional communication is required for obtaining grid based field variables from other ranks to perform interpolation.

The contributions presented in this paper are as follows. Firstly, the computational imbalance due to particle update arising from an uneven distribution of particles in a turbulent flow is quantified. Secondly, the cost of the data transfer necessary to restore load balance by distributing the particles independently of the grid is studied. This is done in the context of realistic particle diffusion rates in turbulent flows and a strong scaling study is performed to a maximum of 32,000 compute cores. Strong scaling to large number of compute cores on a state of the art petascale machine, gives rise to particle densities per rank that is representative of current production parti- 
cle simulations on emerging multi-petaflop machines and anticipated exascale architectures. Thirdly, the overhead from the global communication necessary for bookkeeping and the effectiveness of alternate implementations to reduce this overhead and restore performance is quantified. Finally, a hierarchical load balancing algorithm to preserve grid data locality by generating a balanced particle distribution is outlined. This algorithm also includes flexibility to address many-core nodes by allowing a hierarchical distribution that can preserve locality at several levels.

\section{Related work}

The spatial domain decomposition based algorithm has been extensively used in production particle-in-cell (PIC) hybrid and molecular dynamics solvers $[16,17,18,19]$. The general concurrent particle in cell (GCPIC) algorithm [5] has been used for particle in cell plasma simulations [20] where the grid partitions are adjusted in such a way that they contain equal number of particles. This algorithm does not ensure equality of grid points for the PDE solve and is used when particle computations are more expensive than grid calculations. The approach where particles are equally shared among all compute cores is seldom used due to the overhead associated with restricted data locality and increased data transfer for cases where particles are scattered throughout the computational domain. The question of whether restricted data locality will be offset with better load balance of particles among all MPI ranks remains unanswered and an efficient paradigm to balance both data locality and particle load balance is studied here. The spatial as well as particle sharing algorithm has been compared by Qiang et al. [21] 
in the case of particle beam PIC simulations. The particle sharing algorithm exhibited better strong scaling compared to the spatial algorithm for a maximum of 32 MPI ranks. A more extensive comparison study is performed here using 1000s of compute cores, which is relevant in emerging petascale and exascale architectures. In both the techniques discussed above, ranks tend to have only one sided information. For instance in the spatial partitioning method, every rank has the information about the data to be sent regarding the number of particles that are leaving its domain to the receiving MPI ranks. But the receiver is uncertain about the amount of data coming and which rank it is from. In the particle sharing algorithm, every MPI rank has the information about the grid partition its particles belong to and the amount of data it needs to receive from the grid partition. But the rank owning the grid partition is unaware of the grid data it needs to send and to which rank. Therefore, a bookkeeping step is required prior to actual data transfer in both the techniques which is typically achieved through collective communications such MPI Allgather or Alltoall. These collective communication methods return arrays whose length is proportional to the total number of ranks and hence tend to be costly when used with large number of MPI ranks $[22,23]$. The use of collectives in this scenario also results in the bookkeeping of null information regarding no exchange between two MPI ranks. The use of one sided remote memory access (RMA) communication paradigm in the MPI-2 standard can circumvent these problems and its performance pertaining to particle tracking is studied here. The use of one sided communication has been previously studied for Monte Carlo particle codes [24] and is seen to scale well on multiple compute cores. The use of RMA 
and Partitioned Global Address Space (PGAS) languages on hardware with support such as the "Gemini" interconnect have been previously studied to reduce communication costs for large number of small messages [25]. A factor of 5-10 times increase in effective data transfer rate for data sizes on the order of $8 \mathrm{~B}$ to $2 \mathrm{kB}$ has been reported using one sided communication as opposed to two sided message passing.

Dynamic load balancing of PIC simulations using a taskfarm approach have been studied by Othmer et al. [26] where a master MPI rank distributes tasks to other ranks as soon as they are finished with their work. The slave ranks have to wait in a loop to receive tasks which includes transfer of both particle and grid data.

Load balancing strategies for N-body simulations such as in astrophysics have been implemented through Orthogonal Recursive Bisection (ORB) techniques $[27,28]$ which partition the domain recursively along each direction leading to a tree data structure. ORB have been successfully used along with particle algorithms such as fast multipole methods (FMM) and Barnes Hut (BH) algorithm for effective load balancing. N-body solvers involve particle to particle interactions and do not involve particle mesh interactions which is the focus of the current study.

Octrees and space filling curves [29, 30] have been used in Adaptive Mesh Refinement (AMR) PDE solvers for load balancing the number of grid points per MPI rank. An octree data structure is created for each level of refinement and a space filling curve is used to traverse the leaves of the forest and partitioned appropriately to balance load. These are single objective load balancing methods which do not involve both particles and particle mesh in- 
teractions. They greatly differ from combined particle-grid systems because the two are mutually orthogonal entities and load balancing has to happen over both particle and grid domains with minimal data movement. It might be possible to adapt these approaches for a hybrid particle mesh problem, but this has not yet been done and is not the focus of this work. The use of Inverse spacefilling partitioning (ISP) have also been explored by Pilkington and Baden [31] for PIC solvers, which give rise to balanced particle and grid workload on non-uniform domain partitions. This partitioning, although computationally well balanced, can be detrimental to codes that require higher order accuracy for field calculations, typically required for particleladen turbulent flows. For instance, turbulent combustion code, S3D [32], requires 4 layers of ghost grid-points per rank to obtain an 8th order accurate stencil, which is easily obtained from a regular cartesian decomposition. Additional bookkeeping and overhead will be incurred to obtain ghost grid data for non-uniform partitions with irregular boundaries, when halo points could span partitions of multiple processors.

Load balancing methods involve the equalization of work load among all MPI ranks at a given point in the simulation. The loads can be abstracted as a discrete function of the rank. The work transfer vector, that represents the amount of work that needs to be sent or received by a MPI rank to restore load balance, can be obtained using hierarchical load balancing [33], generalized dimensional exchange [34] or diffusion type methods [35]. Hierarchical methods for load balancing involve splitting the group of ranks into two groups recursively and balancing the load among them. These methods have an $\mathrm{O}(\mathrm{N})$ worst case convergence ( $\mathrm{N}$ is the total number of MPI ranks) 
which has been proved analytically by Willebeek-LeMair et al. [33]. The load balancing method described in this study (Section 4.1.3) can be viewed as a modification of the hierarchical method that equalizes work while preserving locality of data among ranks that are local to each node of the supercomputer. The generalized dimensional exchange method pairs ranks in each dimension of a 3D torus or a hypercube processor network and equalizes their loads by sending and receiving appropriate amount of work load from each other. Diffusion type methods involve a discrete Laplacian smoothing of the load function which leads to equalization with a worst case convergence on the order of the total number of ranks. These algorithms have been implemented in third party libraries such as SCPLib (Scalable Concurrent Program Library) [36] and used with particle simulations. Substantial performance enhancement through the above mentioned load balancing methods have been achieved for single objective calculations such as Direct Simulation Monte Carlo (DSMC) simulations which involve particle movement and particle particle collisions. PIC solvers which involve bi-objective calculations with coupled grid based solves and particle movement have difficulty achieving parallel efficiency [34].

\section{Motivation}

This work involves the development of a proxy application for the particle solver in the turbulent combustion code S3D [32]. S3D solves the multispecies compressible Navier Stokes equations with finite rate chemistry on a domain decomposed Cartesian grid infrastructure. The fluid equations are coupled with a particle model for spray combustion applications [13]. Particle 
(a)
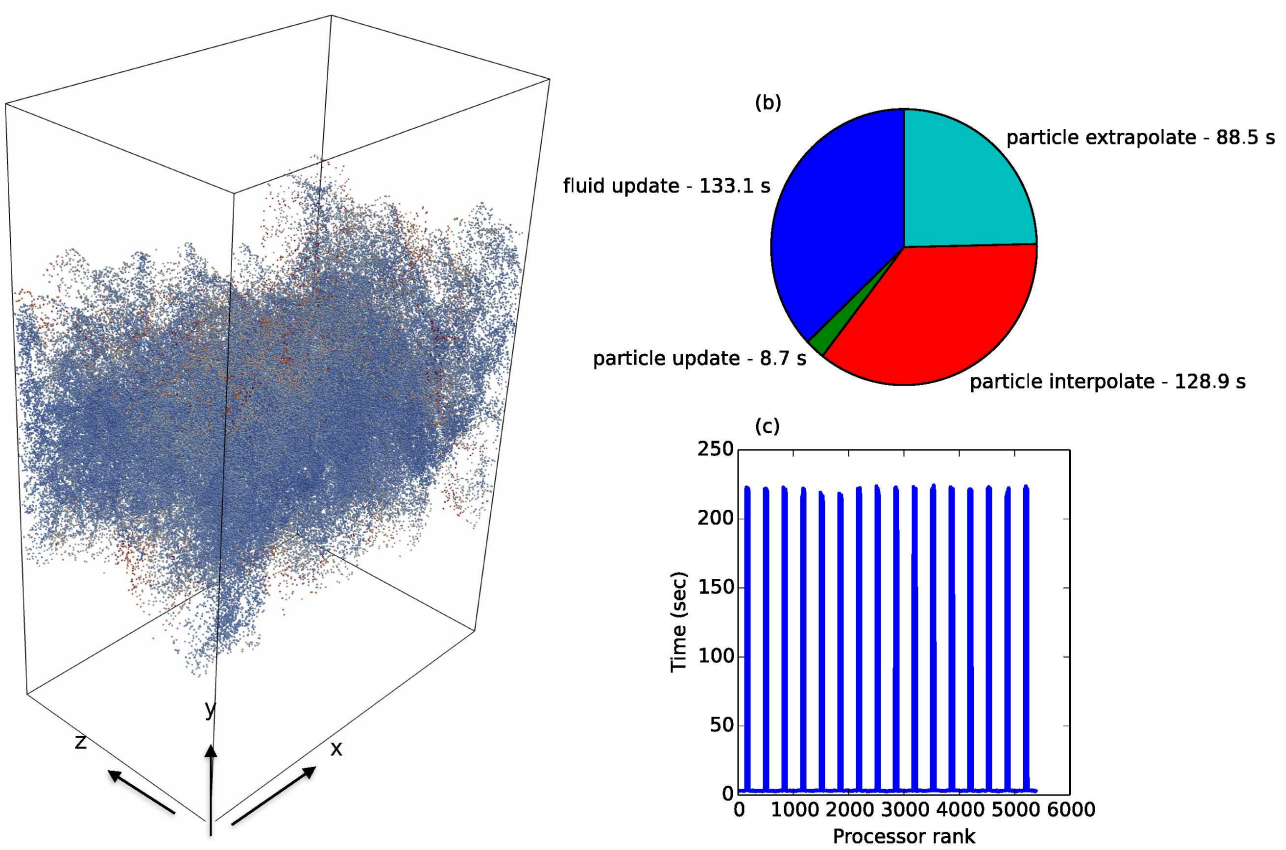

Figure 1: (a) Snapshot of particles for a typical S3D run, (b) pie chart depicting the split of total simulation time and (c) plot of particle solver time for each MPI rank.

updates are performed using interpolated fluid quantities (fluid velocity, temperature and species concentrations). Momentum and energy source terms from particle position and quantities are extrapolated back on to the grid and used in the fluid solution. The parallel decomposition of particles is done using a spatial algorithm where the particles are co-located with the grid partitions. This decomposition leads to two problems. Firstly, the load imbalance in particle calculations due to uneven distributions and secondly the uneven allocation of large amounts of memory on compute cores that own large number of particles. A snapshot of particles for a typical S3D run on Edison supercomputer [37] for a particle laden turbulent jet is shown in Fig. 1(a). The global cartesian grid in this example has 125 million points with 
576, 504 and 432 points along $\mathrm{x}, \mathrm{y}$ and $\mathrm{z}$ directions, respectively. The total number of particles used in this simulation is 80 million. The MPI spatial decomposition for this case uses $24 \times 14 \times 16$ ranks along $\mathrm{x}, \mathrm{y}$ and $\mathrm{z}$ directions, respectively. Figure 1(b) shows the time taken by various update stages of the algorithm over 100 time steps. The particle update time is more than half the total simulation time, with interpolation of grid data onto particle positions being the most expensive operation. Figure 1(c) shows the time taken by the particle solver (includes particle move, particle interpolation and extrapolation) by each compute core. There is significant imbalance in the time taken by each core due to the varying number of particles in each grid partition. It is also seen that only one seventh of the total number of ranks (768 in this case) actually contain particles and hence takes significantly larger computation time compared to other ranks. Clearly, a spatial decomposition of particles co-located with the grid leads to sub-optimal utilization of compute cores. Since particle update, interpolation and extrapolation are operations that depend heavily on the particle count, a strategy where particles are shared equally among all ranks will lead to efficient use of all compute cores at the cost of the loss of data locality, where grid data for interpolation need to be moved to rank that owns the particles. The time for grid data movement, where every grid partition that contains particles sends its entire grid data to ranks who do not have particles, is plotted for varying number of core counts in Fig. 2 along with the time taken by the particle solver. The former tends to increase with larger core count due to an increase in the number of communication events as well as the use of collective operations over larger communicator size. The particle solver times scale with increase in the 


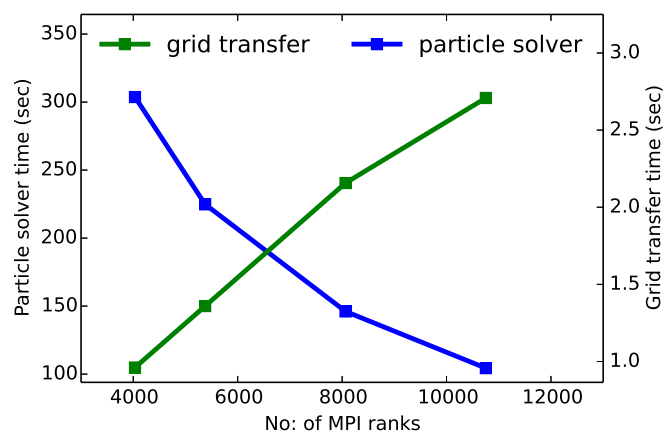

Figure 2: Strong scaling of S3D particle solver for varying number of compute cores along with the time taken for grid transfer plotted on secondary y axis.

number of ranks. If the particles were to be shared equally among all MPI ranks, the solver time shown in Fig. 2 further reduces by a factor of 7 and the time for grid data communication becomes close to about $18 \%$ of the particle solver time. Therefore, grid communication time becomes significant when particles are shared equally among all compute cores, and an intelligent particle partitioning where grid data locality is maximized becomes important. We expect the trend that the cost of data motion and importance of carefully balanced particle decomposition will continue through near term and future architectures. Table 1 indicates the approximate average particle densities per core that would result from moving the "institutional scale" test case to a selection of current and anticipated future leadership class compute facilities. Of note, such a strong scaling exercise results in particle and grid point counts per rank several orders of magnitudes smaller than the baseline case, leaving little computational effort to hide the latency of even fully overlapped communication strategies. We believe that strong scaling is a good metric for exascale design in combustion DNS simulations because an order of 1000 change in computing power will affect the Reynolds number resolution only 
by a factor of $10^{4 / 3}=21.5$. This factor increase will not get us closer to doing realistic high Reynolds number flow simulations. It will then be more practical to investigate how current simulations (that have runtimes on the order of several days) can be run faster with 1000 times computing power. Some of the canonical configurations of interest simulated by the S3D community, are already limited by strong scaling. For example, the homogenous charge ignition study by Bhagatwala et al. [38] involved a grid size that could only be expected to have good performance on $\mathrm{O}(20 \mathrm{k})$ computational cores and would be impossible to run with S3D on more than $\mathrm{O}(130 \mathrm{k})$ cores due to technical limitations in the code.

We can however observe that several computational problems of interest can be done with sufficient resolution at the petascale and the promise of exascale is the ability to incorporate new physics and richness of interacting processes. For example, the Jet in cross-flow case simulated by Grout et al. [39] could be repeated for a liquid fuel involving spray droplets as opposed to higher Reynolds number, and in our opinion this would be the more scientifically interesting path forward. Also of note, the ratio of the degree of expected coarse grain parallelism between the institutional computing baseline and a current leading machine such as Edison is approximately the same (a factor of 10-30) as between that machine and an anticipated Exascale

\footnotetext{
${ }^{1} 1$ 'scaleable unit' of Peregrine, hosted at NREL

${ }^{2}$ Cray XE6 located at NERSC

${ }^{3}$ Cray XC30 located at NERSC

${ }^{4}$ Cray XK7 located at NCCS, 1 rank per GPU

${ }^{5}$ Cray XK7 located at NCCS, 1 rank per Opteron core

${ }^{6}$ Approximate size of future NERSC [40]

${ }^{7}$ Scale up of Titan to Exascale level guided by 'fat node swim lane' [41]
} 
Table 1: Grid point and particle densities per MPI rank resulting from running particle jet test case on a selection of current and anticipated future computing resources in strong scaling paradigm.

\begin{tabular}{|c|c|c|c|c|c|}
\hline Machine & Nodes & $\begin{array}{l}\text { MPI Ranks } \\
\text { per node }\end{array}$ & $\begin{array}{c}\text { Coarse grain } \\
\text { parallelism }\end{array}$ & $\begin{array}{l}\text { Gridpoints } \\
\text { (per rank) }\end{array}$ & $\begin{array}{c}\text { Particles } \\
\text { (average/rank) }\end{array}$ \\
\hline Baseline $^{1}$ & 224 & 24 & 5376 & 23328 & 14880 \\
\hline Hopper ${ }^{2}$ & 6384 & 24 & 153216 & 819 & 522 \\
\hline Edison ${ }^{3}$ & 5576 & 24 & 133824 & 937 & 598 \\
\hline Titan ${ }^{4}$ & 18688 & 1 & 18688 & 6711 & 4281 \\
\hline $\operatorname{Titan}^{5}$ & 18688 & 16 & 299008 & 419 & 268 \\
\hline NERSC-8 (Cori) ${ }^{6}$ & 9000 & 60 & 540000 & 232 & 148 \\
\hline Exascale SW-2 ${ }^{7}$ & 100000 & 10 & $1 \mathrm{M}$ & 120 & 77 \\
\hline
\end{tabular}

machine. The anticipated degree of coarse grain concurrency on the exascale machine was achieved by extrapolation of the 'fat node' exascale swim lane identified in 2009 in the report from an US Department of Energy hosted workshop [41]. In that report, a possible prototypical exascale machine was identified as having 100,000 nodes and 10,000-way concurrency within a node. An alternative path using 1,000,000 nodes and single-way concurrency within a node was also identified, although that appears less likely in the view of systems deployed since the report was written. The Nvidia Kepler GK110 GPU is used today, e.g., in Titan and contains 15 SMX multiprocessors with 64 double precision floating point units each for 960 degrees of parallelism per GPU in the notation of the 2009 report. Scaling this up linearly to 10,000-way concurrency suggests approximately 10 GPUs per node would be consistent with the predicted node size, so we use 10 MPI ranks per node as a representative size. It should be noted that while there is general agreement in the community regarding computing trends supported by products available since the report was written, an exascale machine does not yet exist and 
these comments are only to motivate the work presented here in an approximate context. We assume in these remarks an evolution of the programming environment from 'MPI everywhere' to 'MPI+X', where the ' $+\mathrm{X}$ ' expresses much of the intra-node parallelism leveraged for particle and grid updates, leaving the bulk of computation managed by the 'MPI' programming model related to data movement. For this reason, the proxy application that we will discuss later focusses on the cost of data motion in the MPI context with simple algorithms for particle and grid update.

\section{Particle tracking algorithm}

To understand and tackle the problems of load imbalance and data locality in particle simulations, a stand alone application is developed that serves as a proxy for the full application and implements a particle tracking algorithm with one sided coupling from the grid to the particle solver. The focus here is in the strong scaling limit where a small problem without any cost for grid calculation is used as the test case. This serves as a substitute for a future design case where the grid solution has been well optimized or offloaded to a high-throughput accelerator leaving bottlenecks in the particle mover. The velocity and passive scalar data from a typical turbulent flow simulation on a Cartesian grid is used for the particle tracking code. The grid partitioning used in the turbulent flow calculation is a Cartesian decomposition where each rank is assigned a box.

$$
\frac{d \vec{x}_{i}}{d t}=\vec{V}\left(\vec{x}_{i}\right)
$$


Every $\mathrm{i}^{\text {th }}$ particle is moved using interpolated velocities using Eq. 1 where $\vec{x}_{i}$ is the position of the particle and $\vec{V}$ is the interpolated velocity at the particle's location. The particle data structure contains three double precision numbers that store its coordinates along with a chosen number of double precision passive scalars that store interpolated values from the grid. It also contains two integer variables indicating the grid partition that the particle belongs to and a variable that stores the escape status of the particle. Particles that go out of the computational domain are deleted from the simulation. For the base case simulation results shown below, the number of passive scalars is set to 1 . This case is simplified so as to focus on the imbalance in the number of particles. Most continuum-Lagrangian solvers pertaining to reacting flows include other passive scalars such as species concentrations, temperature and particle size. Therefore, simulations are also done with a typical number of passive scalars seen in combustion simulations. This case will be affected not only by particle imbalance but also by the volume of data transfer. The overall simulation time shown in Fig. 10(c) is for the case with 47 passive scalars, which adds up to 50 double precision numbers per particle. The general algorithm for particle tracking is to first seed the particles at $t=0$, then at each time step identify the grid points that enclose each particle to interpolate the velocity fields to its position and update the particle location using a time stepping method. The interpolation of flow quantities is done using a simple arithmetic averaging. The time stepping method for movement of particles is done using a first order Euler explicit time stepping method. These steps are made simple to focus on the parallel performance aspects and particle load imbalance in typical simulations. 


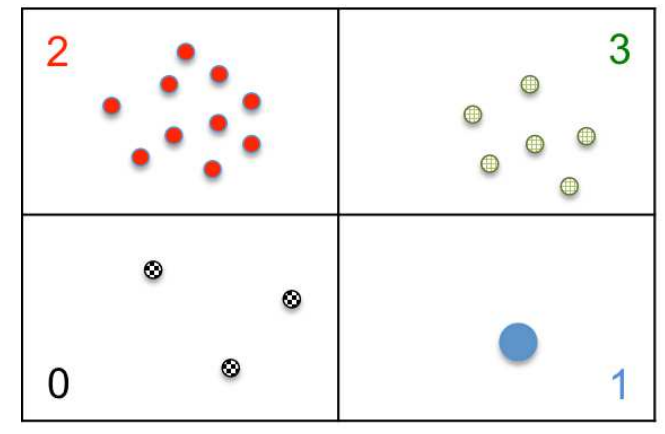

(a)

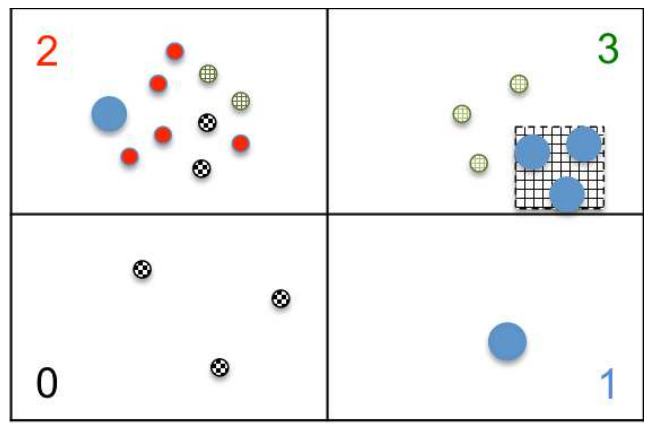

(b)

Figure 3: Schematic of (a) the spatial algorithm and (b) particle sharing algorithm. Particle pattern indicates the rank where the particle is local, and the numeric labels indicate the rank where the corresponding grid partition is local.

\subsection{Parallel implementation techniques}

The particle tracking algorithm is implemented using the two commonly used techniques and compared with each other on various performance metrics. The first technique is where all the ranks own particles local to their share of the computational domain, which will be referred to as the spatial algorithm. The second technique is where particles are shared equally among all ranks irrespective of the spatial grid partitions to which they belong. An algorithm to improve the performance of the latter method by increasing grid data locality is also described here.

\subsubsection{Spatial algorithm}

In this technique, particles present in each grid partition are owned by the corresponding MPI rank as shown in Fig. 3(a). The particles are moved using interpolated velocities obtained from the rank's grid partition. After every time step, the data describing particles that escape the boundaries of each grid partition is sent to the rank associated with the new position of 
the particle.

A bookkeeping step is first performed where all ranks send the information about the number of particles that will be transferred to the destination rank. This is typically achieved through an MPI_Alltoall collective communication, if particles are assumed to cross over to any grid partition depending on the direction of velocity and time step size. The overhead of MPI_Alltoall call on large number of ranks can be mitigated by re-implementing the collective using one-sided remote memory access communication paradigm. Alternatively, the particles can be assumed to move to ranks that are nearest neighbors to the grid partition for relatively small time steps used in the experiments in this study. This will require a bookkeeping step with only point to point communications. A comparison of the three different bookkeeping methods for this algorithm is studied in section 5 .

After this step, every rank knows how many particles are to be received from each grid partition and is able to allocate an appropriate amount of memory for the receive buffer. This algorithm closely follows the parallel particle tracking algorithm used for diagnostics in turbulent reacting flow codes such as S3D $[32,42]$. The pseudocode for this method for a single time step is described in algorithm 1. The memory requirements per MPI rank will predominantly consist of the number of particles that each grid partition owns, which can be a large fraction of the total number of particles for an uneven distribution. For instance, the isotropic turbulent box simulations shown in Fig. 5 would require the storage of all particles on one rank, which accounts for memory usage on the order of 4 GB for 10 million particle data structures with 50 scalars. The grid data storage will depend on the number 
of grid points per rank which is typically small and remains constant over the simulation.

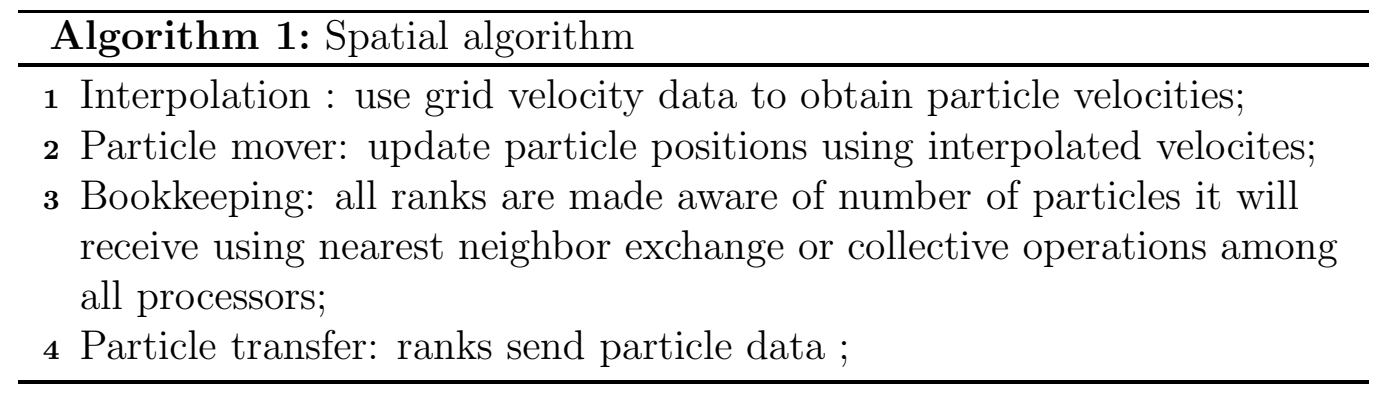

\subsubsection{Particle sharing algorithm}

The particle sharing algorithm divides the particles equally among all ranks, regardless of the associated grid partitions they belong to, as shown in Fig. 3(b). Every rank can calculate the grid partitions necessary to update its particles using the particle coordinates as well as the grid spacing in the uniform Cartesian grid. Every rank therefore needs field information for particles that are not local to its grid partition. The non-local field data is sent to each rank in the form of a coordinate axis-aligned bounding box. An example bounding box for particles owned by rank 1 present in the grid partition of rank 3 is shown in Fig. 3(b).

A bookkeeping step is also required here, where the extents of the bounding box are sent to the rank that owns the corresponding grid partition. This step gives every rank the information to allocate its send and receive buffers for the incoming/outgoing field data. This step needs a collective operation like an MPI_Allgather or MPI_Alltoall, which can prove to be expensive when used with large number of cores. These collective operations can be reimplemented using one sided remote memory access (RMA) communication 
in the MPI-2 standard [43], which tends to cost the same as point to point communication using MPICH2 library (on supercomputer Hopper used in this work). In the remote memory access technique, every rank allocates a double precision array that is of size equal to six times the total number of ranks. The six double precision values are the three dimensional coordinates of two opposite corners of the box. Every MPI rank who needs to send the extents of the bounding box does a one sided communication using MPI_Put into the array allocated by the receiving grid partition rank. Appropriate offsets are also provided which is equal to six times the sender rank here. The non-scalable collectives can be also be re-implemented using scalable MPI_Allreduce call which updates the number of receives per rank. This information can be used in a loop with MPI_Recv with MPI_ANY_SOURCE as the source rank. Bookkeeping with collective operations tend to gather information that may not be necessary for a given MPI rank. For instance, a rank that does not have to send any grid information to any other rank also participates in the gather/reduce process where null values are sent and received, which can be avoided using one sided communication.

After this,the actual send and receive of field information from the grid partition is initiated. These steps are shown in algorithm 2 for a single time step. The bounding box, which may be compact initially, could eventually become very large over time. For example, in the case of homogeneous isotropic turbulence (Fig. 5 (a)) the particles are spread out in the computational domain due to mean turbulent diffusivity. The particle data stored per rank is typically smaller compared to the spatial algorithm and remains more or less constant with small variations due to escape of particles at phys- 
ical boundaries of the computational domain. The grid data storage varies over time due to the size and number of bounding boxes required by each rank, unlike the spatial algorithm. For instance, the turbulent jet simulations shown in Fig. 11 when run on 800 ranks with 1.6 million grid points per rank, may require an average of $65 \mathrm{MB}$ of data per rank for a case of 4 scalars per particle. This will translate to about 1 GB for a case of 50 scalars.

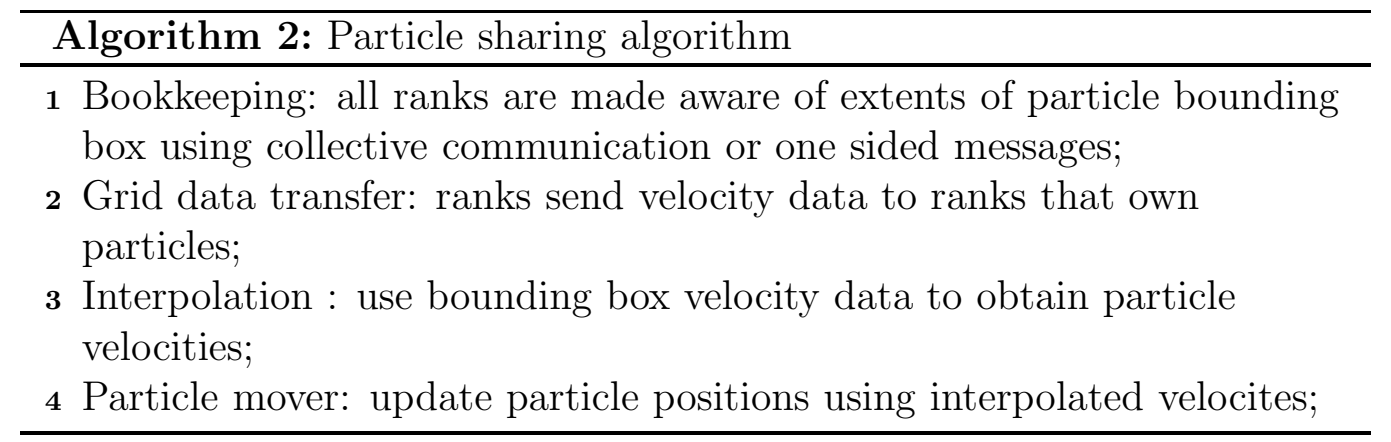

\subsubsection{Locality Preserving equalization for particle sharing algorithm}

To deal with the unfortunate tendency of the bounding boxes for a set of particles to grow over time, it is necessary to rebalance the particles in a way that respects particle clustering, data movement and memory costs. This can be achieved by increasing locality in the sense of overlap between the necessary bounding box of data and the local grid data. To that end we propose an equalization strategy where particles are first spatially decomposed and then redistributed only as necessary to balance the number of particles per rank. The aim of the equalization algorithm is to distribute particles among ranks in such a way that a large fraction of particles are local to the rank's grid partition. The starting point of the algorithm is the spatial decomposition of particles where each rank owns particles that are local to 


\begin{tabular}{|c|c|c|c|c|c|c|}
\hline Step 0 & 2 & 10 & 50 & 5 & 30 & 22 \\
\hline Step 1 & 26 & 10 & 26 & 5 & 30 & 22 \\
\hline Step 2 & 26 & 10 & 26 & 18 & 17 & 22 \\
\hline Step 3 & 18 & 18 & 26 & 18 & 17 & 22 \\
\hline Step 4 & 18 & 18 & 21 & 18 & 22 & 22 \\
\hline Step 5 & 20 & 18 & 21 & 18 & 20 & 22 \\
\hline Step 6 & 20 & 20 & 21 & 18 & 20 & 20 \\
\hline Step 7 & 20 & 20 & 19 & 20 & 20 & 20 \\
\hline
\end{tabular}

Figure 4: Equalization of an array with six integers.

its grid partition. The spatial decomposition of particles creates imbalance in the number of particles per rank. The equalization algorithm works as shown in Fig. 4, where an example case for six ranks with varying number of particles, depicted as an array of six integers is shown. The equalization can be done by finding the maximum and minimum of the array (indicated by boxes) each step and equalizing the two numbers to their averages. The rank with the maximum number of particles sends the appropriate number to equalize with the rank having minimum number of particles. The worst case convergence of the algorithm is $O(N)$ where $N$ is the total number of MPI ranks. Particles tend to be distributed in such a way that a larger number of particles remain local to the grid partition at the end of equalization. Similar algorithms have been used in load balancing Monte-carlo simulations [44] with $O(\log N)$ convergence, where particle-grid interactions were not 
expensive and grid data had been replicated over all processors.

The equalization algorithm for a large number of ranks on the order of 1000 can be done efficiently by grouping set of ranks together. The grouping is done in such a way that each of them contain ranks that reside on each node in the supercomputer thereby ensuring nodal locality. The equalization iterations are performed within each group interleaved with a few coarse iterations across the groups. The algorithm can be split into 3 different steps. The first step is to obtain an integer array containing number of particles per rank after spatially redistributing particles as shown in algorithm 3 . This is done using one sided MPI_Put and MPI_Bcast, instead of non-scalable MPI_Allgather call. MPI_Bcast is a scalable operation only when the message size is fixed, unlike the situation here where the size is proportional to the number of processors. However, we have observed that the combination of MPI_Put and MPI_Bcast is more efficient than MPI_Allgather on large number of MPI ranks. The second step is to perform the equalization iterations as shown in algorithm 4 . Two integer stacks per processor containing rank and corresponding particle count are used to record the particle movement. The stacks corresponding to the rank containing maximum number of particles is popped until the required number for equalization is removed. The appropriate number of particles and corresponding rank is then pushed to the stacks corresponding to the rank containing minimum number of particles. These steps are performed by all processors on the global integer array from algorithm 3 and does not involve any communication. The information in the two stacks are then used for point to point communication of particles, as shown in algorithm 5. This algorithm is a modified version of the 


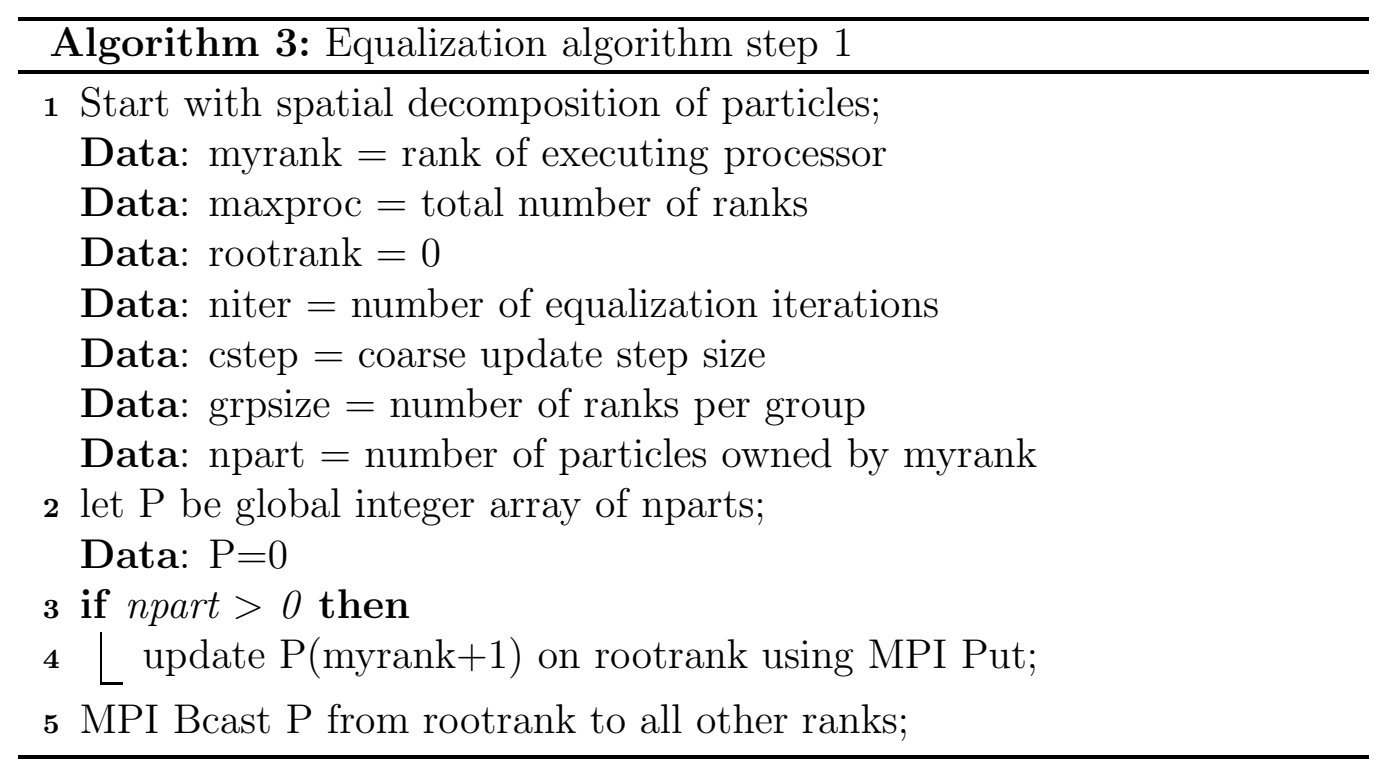

hierarchical load balancing method $[33,34]$ which preserves nodal data locality by appropriately grouping the ranks. This method is also compatible with parallel paradigms where compute nodes have multiple MPI ranks with OpenMP threading done within each MPI process.

\section{Performance comparisons and results}

The performance of the different parallelization algorithms described in section 4.1 is studied using two experimental problems with different communication and execution characteristics. These are as follows.

1. Particle tracking in an isotropic turbulence box where particle count is significantly greater than number of grid points. Here the imbalance in particles per rank significantly affects performance.

2. Particle tracking in a turbulent jet where the number of grid points vastly exceed the number of particles. Here the communication of grid 


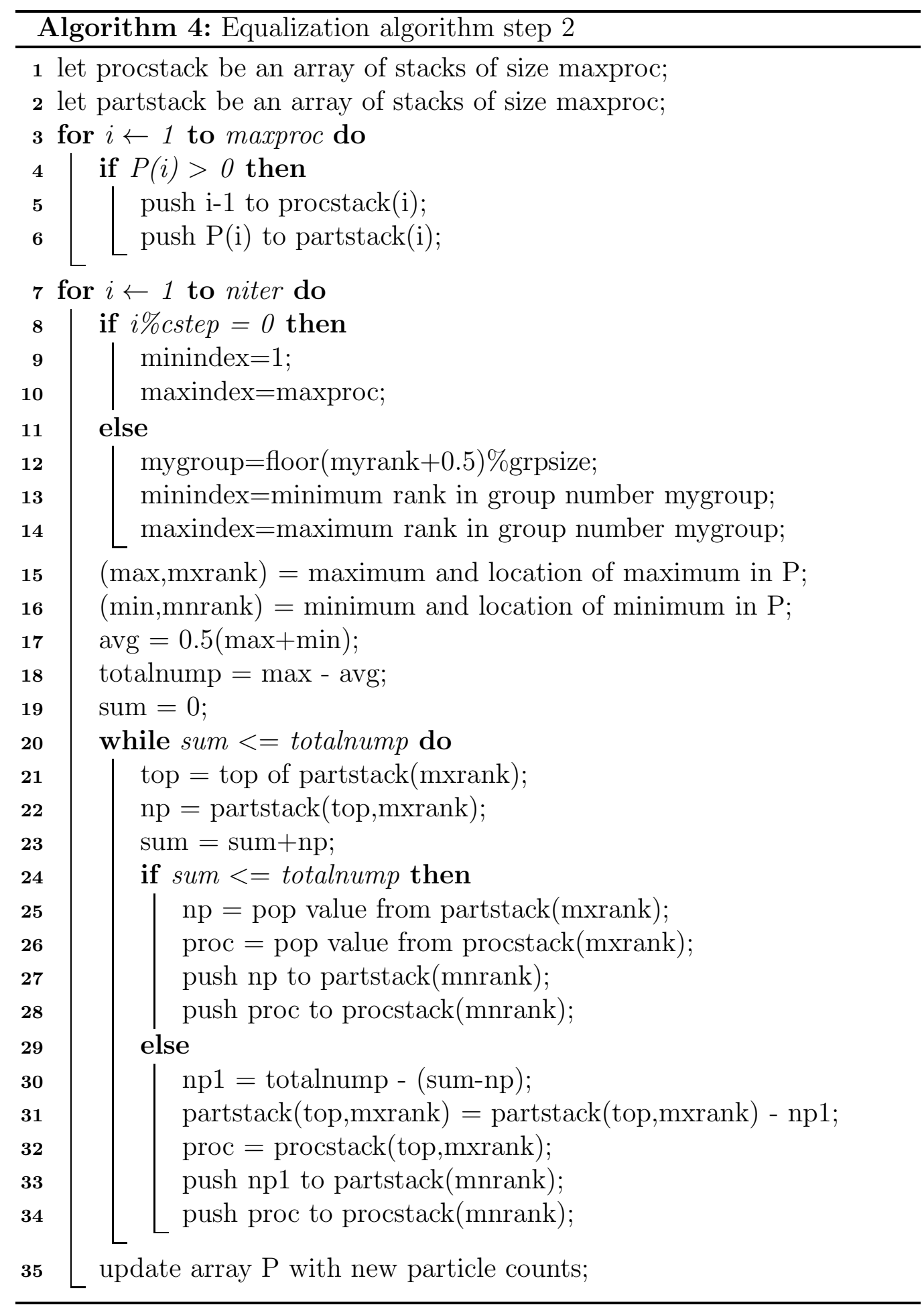




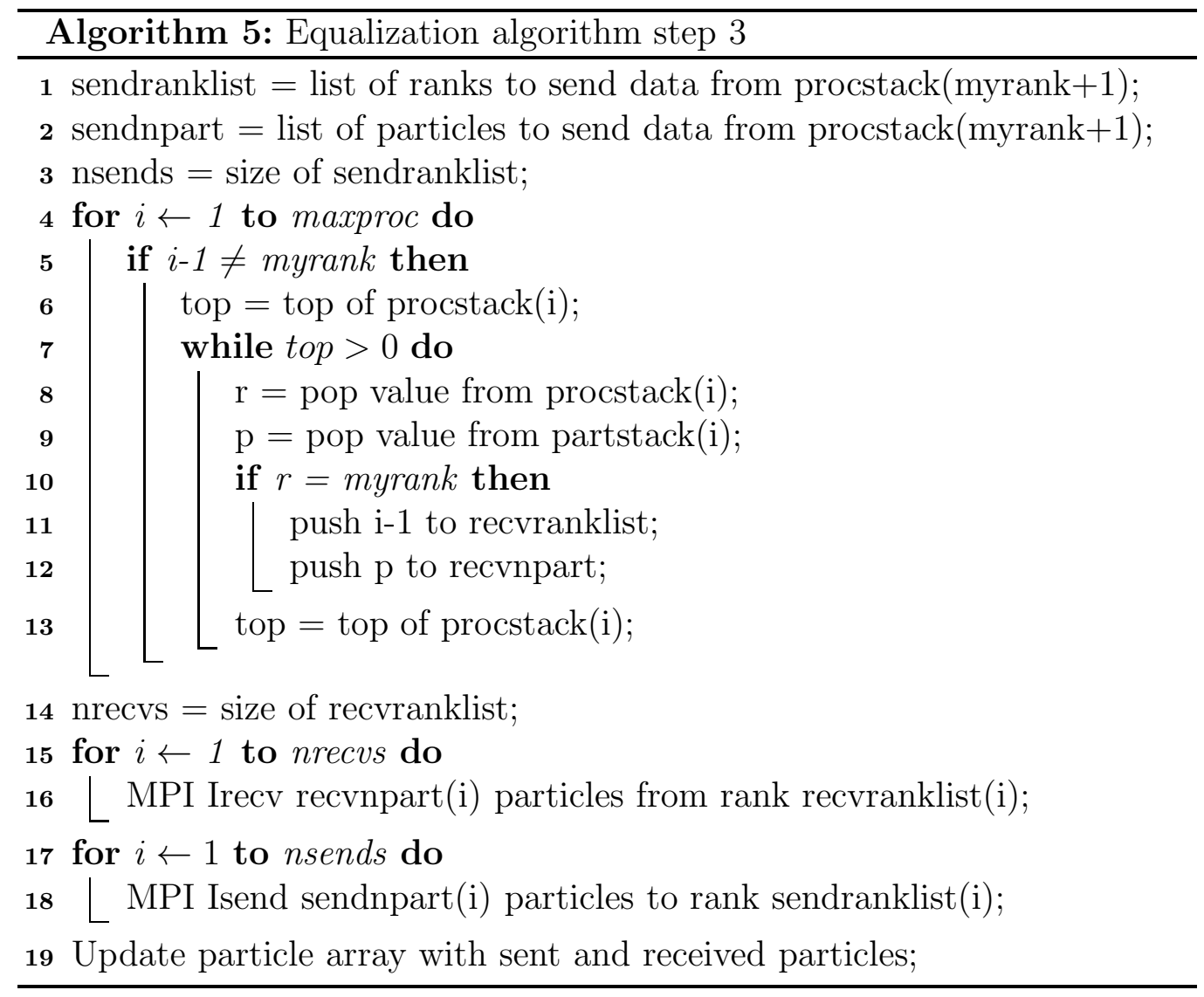


data becomes expensive and efficient strategies to balance particles while maintain greater data locality is dealt with.

The two experiments studied here focus on imbalance in execution costs and importance of data transfer costs, respectively. Both of these play an important role in determining performance when current petascale simulations are strong scaled to exascale.

These simulations are performed on Cray XE6 supercomputer Hopper [37] which has 24 AMD cores (clock speed of $2.1 \mathrm{GHz}$ ) per node. Hopper has a total of 153,000 compute cores with peak performance of 1.28 petaflops. The largest strong scaling run performed in this study uses 32,768 cores which is about $20 \%$ of the total number of cores on Hopper. The MPI implementation used is MPICH2 [45] and the compiler used is the default Portland Group Inc. (PGI) fortran compiler [46].

\subsection{Particle tracking in isotropic turbulence box}

The parallel performance of the spatial as well as the particle sharing algorithm is compared for the homogeneous isotropic turbulence box problem. The computational domain is a cube of dimension $L=1 \mathrm{~m}$ with 64 points along each direction, as shown in Fig. 5. The Cartesian domain decomposition for a case of 64 ranks is shown here with 4 ranks along each of the coordinate directions. The grid partitions are assigned to ranks one after the other in a three dimensional matrix with the $\mathrm{x}$ coordinate being the quickest index. Particles are located in a cube with dimension $L / 4$ at time $\mathrm{t}=0$ as shown in Fig. 5(a). The velocities in the isotropic turbulence box are on the order of $3-4 \mathrm{~m} / \mathrm{s}$ and the time step for particle position update is 

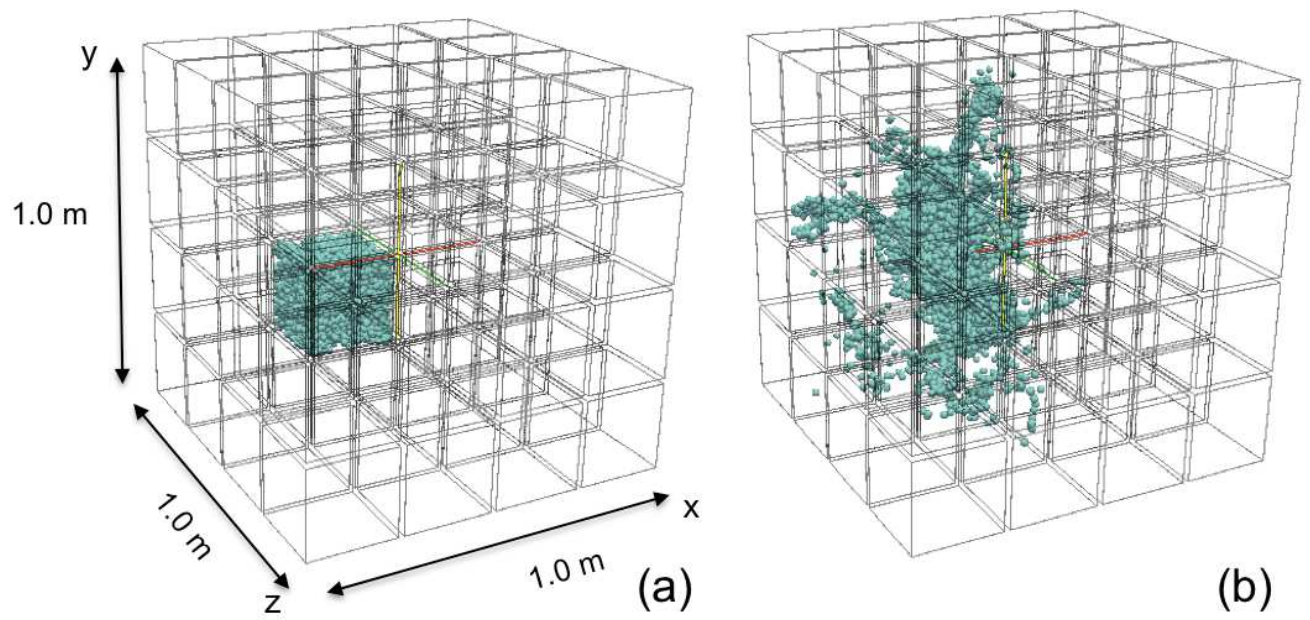

(b)
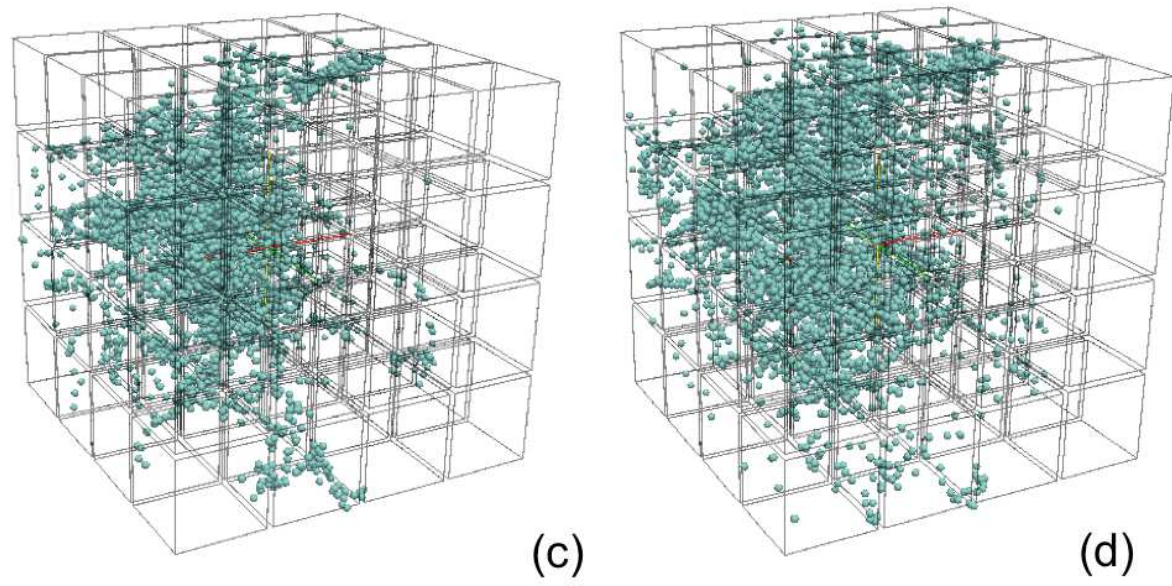

Figure 5: Particle transients for the homogeneous isotropic turbulence box at time (a) $t=0$ ,(b) $\mathrm{t}=0.3$, (c) $\mathrm{t}=0.6$ and (d) $\mathrm{t}=1$ second, run on 64 compute cores. 
Table 2: Parameters relevant to execution load and data transfer for the isotropic turbulence box problem

\begin{tabular}{|c|c|}
\hline Parameters & Value \\
\hline Total number of grid points & $2.62 \mathrm{E}+05$ \\
Total number of particles & $1.0 \mathrm{E}+07$ \\
Number of particles per grid point & 38 \\
Number of time steps to move one grid cell & 4 \\
\hline
\end{tabular}

assumed to be 1 millisecond. Fig. 5(b), (c) and (d) show particle transients at time $\mathrm{t}=0.3,0.6$ and 1 second. It is seen that the particles spread out due to turbulent mixing and eventually percolate into all grid partitions. The initialization of particles in the domain is done here in a such a way that it becomes the worst case scenario for either of the algorithms. For the spatial algorithm, there is an imbalance in the number of particles per rank. The particle sharing algorithm will require data transfer among all ranks because of the loss of grid locality of particles. The computational aspects relevant to execution load and data transfer for this problem are presented in Table 2. The number of time steps required to move one grid cell shown here, is an estimate from average fluid velocity and grid spacing. The number of grid points moved per time step will be different and can evolve over time, depending on the position of the particle. The comparison study of the two algorithms is performed using 10 million particles for 500 time steps. The number of ranks along each direction is varied from 4 to 32 , doubling each time. The study is thus done for 64, 512, 4096 and 32,768 MPI ranks. Fig. 6 compares the time required for particle move and interpolation step for both the algorithms. The time taken by each rank for the spatial algorithm tends to be imbalanced due to clustered distribution of particles compared to the 

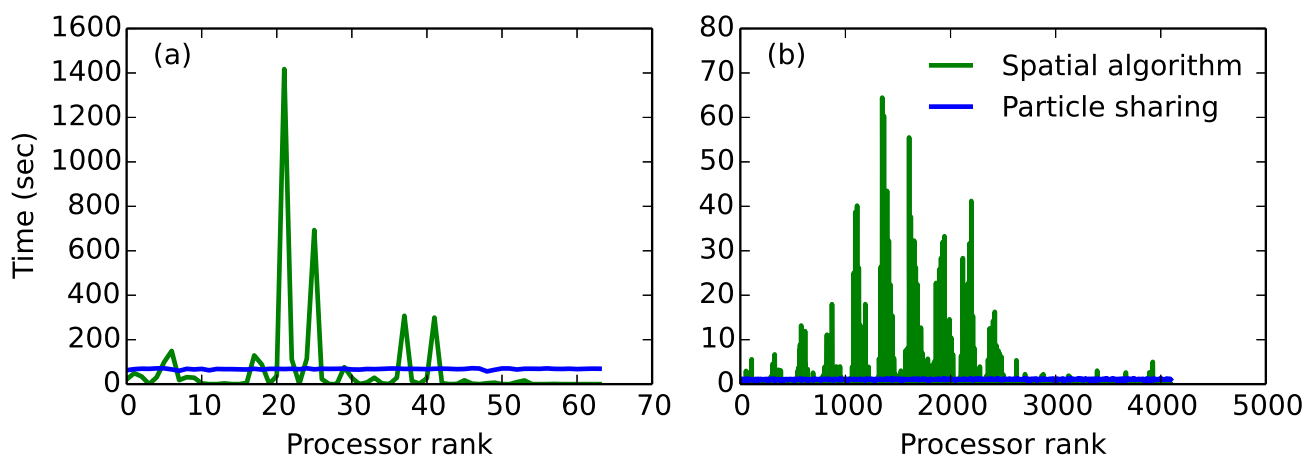

Figure 6: Comparison of particle move and interpolation time for (a) 64 and (b) 4096 core runs using both the spatial and particle sharing algorithm.

particle sharing algorithm. The time taken by the particle sharing algorithm is also seen to reduce by a factor of 64 when run on 4096 cores. The load imbalance in the spatial algorithm would require certain ranks to wait for the ones with significant load to finish before initiating the transfer of particles between ranks. The maximum total waiting time for the spatial algorithm is shown in Fig. 7(a) along with the fraction of the total time spent on waiting by all ranks for varying total number of ranks. It is seen that the waiting time reduces with larger number of ranks because of lesser time required to process lower number of particles. The waiting time fraction is about $96-98 \%$, as shown in Fig. 7(b), which clearly indicates the imbalance in the algorithm even with larger number of ranks.

As mentioned earlier, the particle sharing algorithm requires a bookkeeping step where the extents of the bounding box of the particles owned by a given MPI rank is made known to ranks whose grid partition its particles are present. This requires a collective operation like an MPI_Allgather which can prove to be expensive when used with large number of cores. 

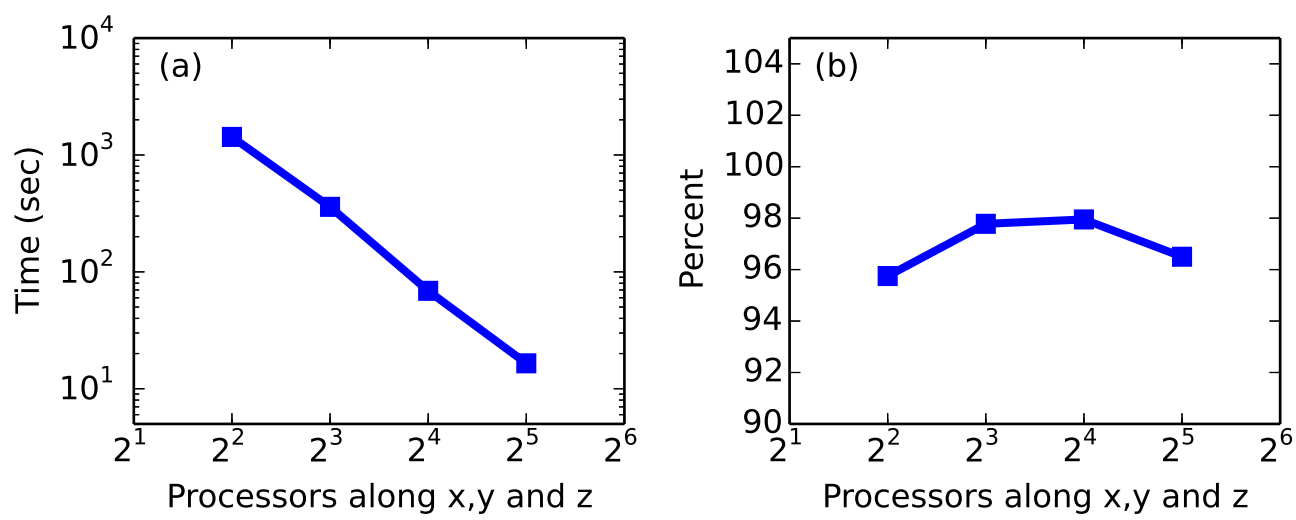

Figure 7: (a) maximum waiting time for the spatial algorithm for varying total number of ranks from 64 to 32,768 ; (b) waiting time fraction for the spatial algorithm.

Table 3: Total simulation time in seconds using MPI_Allgather and MPI_Alltoall to perform bookkeeping for homogeneous isotropic turbulence problem.

\begin{tabular}{|c|c|c|}
\hline communicator size & MPI_Allgather & MPI_Alltoall \\
\hline 64 & 92 & 91 \\
512 & 16 & 14 \\
4096 & 33 & 17 \\
32,768 & 175 & 273 \\
\hline
\end{tabular}


An MPI_Allgather is first done to obtain an array of size equal to the total number of ranks, which contains the number of bounding boxes each rank requires. This information is used to allocate a tight array to hold the bounding box corner information. Then, an MPI_Allgatherv is performed to obtain the extents of the bounding box (coordinates of lower and upper corners of the box which contribute to six double precision numbers) and the corresponding grid partition rank. Alternatively, an MPI_Alltoall communication can be done using an array of size equal to six times the total number of ranks where each rank updates its local array with the extents of the bounding box at appropriate offsets (six times the grid partition rank). The total simulation times using the MPI_Allgather as well as MPI_Alltoall for bookkeeping is shown in Table 3. The times shown are averages of three different runs and did not change appreciably. The time taken for both the collective operations tend to increase simulation time sharply when run using large number of ranks. These results are consistent with benchmarking tests done on this machine [22]. These calls are not scalable and should be carefully used when run on large number of ranks. Fig. 8 shows the fraction of the total simulation time spent doing bookkeeping for varying number of MPI ranks along each direction. For a case of 32 ranks along each direction (translates to 32,768 compute cores in total), it is seen that about $80 \%$ of the time is spent in doing non-scalable collective operations.

The performance of bookkeeping is considerably enhanced by re-implementing the collectives (MPI_Allgather or MPI_Alltoall) using one sided remote memory access (RMA) communication in the MPI-2 standard. Fig. 9 shows the comparison of time taken for performing the bookkeeping operation for 


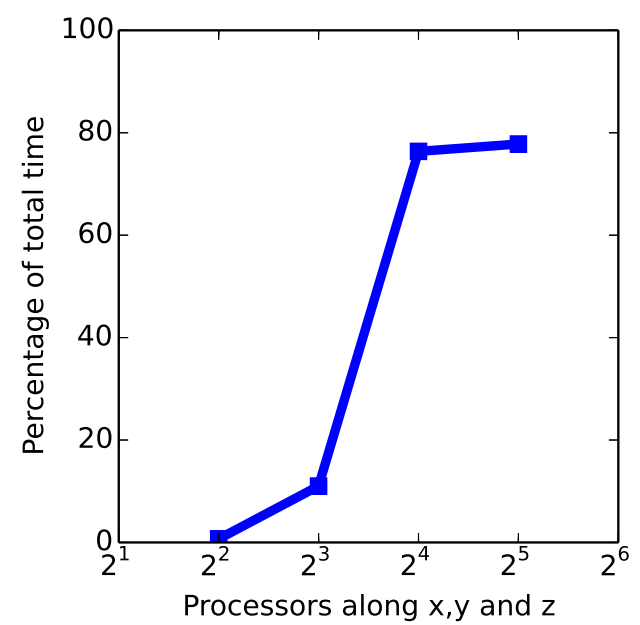

Figure 8: Fraction of time spent by particle sharing algorithm to perform the bookkeeping step using collective operations (MPI_Allgather).
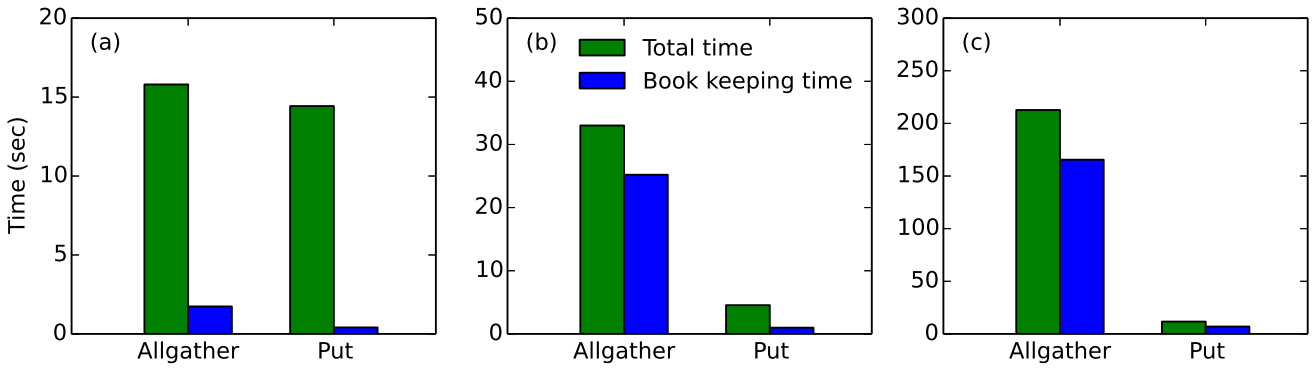

Figure 9: Comparison of the use of MPI_Allgather and remote memory operation "put" for (a) 512 (b) 4096 and (c) 32,768 core runs. 
512 and 4096 core runs, respectively. The effect of the one sided communication is prominent in the case of the 4096 core run where the run time is considerably reduced. Fig. 10 shows the overall simulation time for the spatial algorithm as well as the particle sharing algorithm. The particle sharing algorithm with collective operations tends to perform poorly for large number of core runs and takes longer time compared to the spatial algorithm as shown in Fig. 10(a). The particle sharing technique with RMA is seen to scale well compared to the collective operation method and takes lesser time compared to the spatial algorithm for the case of one passive scalar (Fig. 10(b)) as well as the case with 47 scalars per particle (Fig. 10(c)). The additional number of scalars increases simulation times due to greater number of interpolation calculations done per particle as well as larger data transfer sizes per particle and grid point. Fig. 10(d) shows the total simulation time for the spatial algorithm with three different bookkeeping methods. There is no appreciable change in simulation times except at 32,768 ranks where the scalability issues of MPI_Alltoall becomes important. The one sided reimplementation of the collective and the nearest neighbor method tends to scale better compared to bookkeeping using MPI_Alltoall. The negligible variation in simulation times also imply that the load imbalanced particle execution time dominates over communication.

The strong scaling study shown in Fig. 10 is representative of particle simulations that will be run on an exascale machine with thousands of cores per node. The simulation with largest number of cores in Fig. 10 updates only about 300 particles per rank per time step for the particle sharing algorithm. This will be the situation on an exascale machine for typical particle 

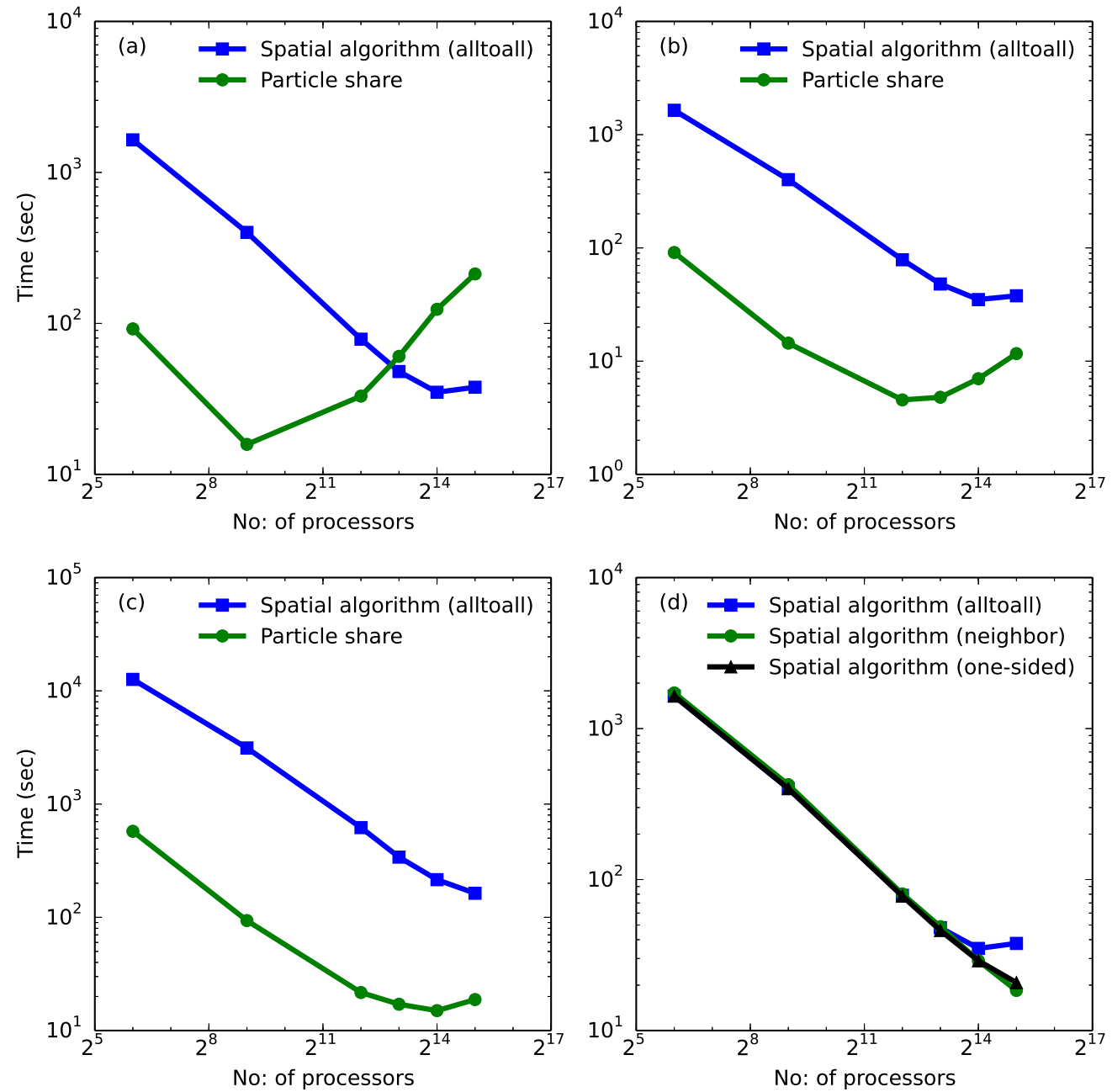

Figure 10: Comparison of total simulation time for the spatial algorithm and particle sharing algorithm with (a) collective gather operation and (b) RMA technique for a case with only 1 passive scalar per particle data structure. (c) shows comparison of spatial and particle sharing algorithm with RMA for a case of 47 scalars per particle data structure. (d) shows the comparison of total simulation time for the spatial algorithm with bookkeeping done using MPI_Alltoall, nearest neighbor and one-sided communications for 1 passive scalar. 


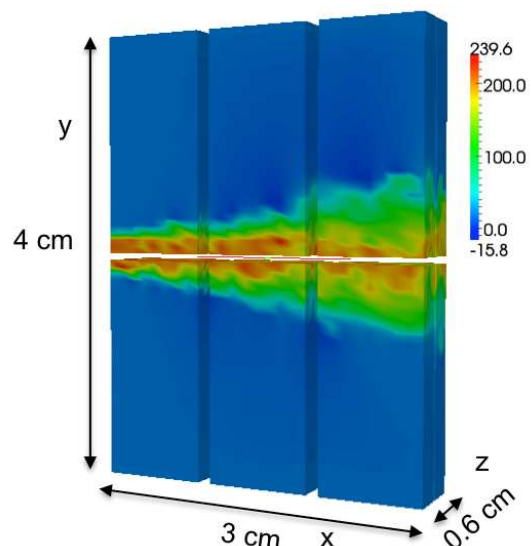

(a)

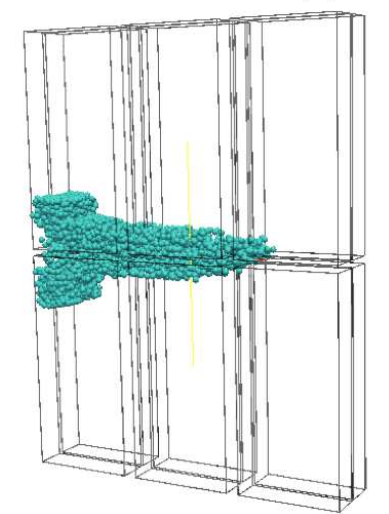

(c)

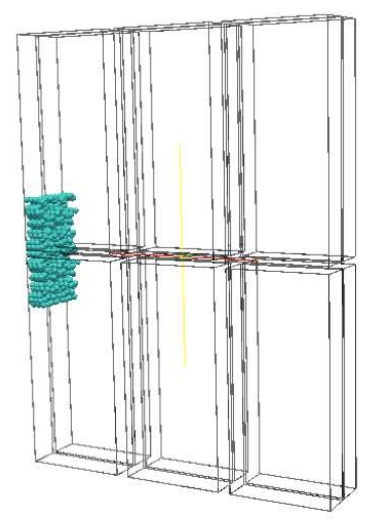

(b)

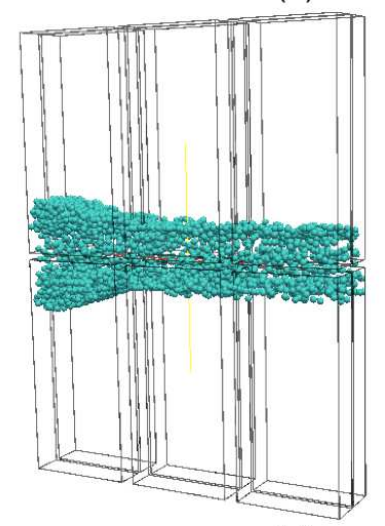

(d)

Figure 11: (a) The dominant component of velocity along $\mathrm{x}$ direction in $\mathrm{m} / \mathrm{s}$ for the turbulent jet case. Particle positions at time (b) $\mathrm{t}=0$ (c) $\mathrm{t}=0.1 \mathrm{~ms}$ and (d) $0.5 \mathrm{~ms}$. Note that particles are seeded in the box-like domain shown in (b) every $0.01 \mathrm{~ms}$.

simulations currently run on petascale machines. The time spent on embarrassingly parallel particle update operations reduces significantly and the overhead of data movement determines total simulation time. This case of increased data transfer with lower particle count is studied in the next section where the number of grid points exceeds the number of particles. 
Table 4: Typical parameters relevant to execution load and data transfer for the turbulent jet problem

\begin{tabular}{|c|c|}
\hline Parameters & Value \\
\hline Total number of grid points & $1.296 \mathrm{E}+09$ \\
Total number of particles & $2.0 \mathrm{E}+06$ \\
Number of grid points per particle & 650 \\
Number of grid points moved per time step & 16 \\
\hline
\end{tabular}

\subsection{Particle tracking in turbulent jet}

A turbulent jet velocity field on larger number of grid points $(2025 \mathrm{x}$ $1600 \times 400$ ) computed from S3D (similar to simulations in ref. [47]) with seeding of particles in regular time intervals is considered here. Fig. 11(a) shows the $\mathrm{x}$ velocity profile and the grid partitioning on 12 MPI ranks for the turbulent jet problem. Fig. 11(b),(c) and (d) show the particle position transients at three different instances in time. The axial velocities are on the order of $200 \mathrm{~m} / \mathrm{s}$ and a time step size of 1 microsecond is used for particle update. Relevant parameters pertaining to execution load and data transfer for this problem are presented in Table 4. As time elapses, the particles are advected most rapidly by the highest velocity near the center of the jet and particles starting in the low velocity co-flow are entrained into the higher velocity region. This results in a highly non-uniform, yet physically meaningful spatial particle distribution.

\subsubsection{Equalization algorithm and load balancing}

The particle sharing algorithm requires the transfer of grid data from spatial partitions, which can become expensive for large number of grid points. The spatial algorithm on the other hand preserves particle grid locality but creates load imbalance of particles. Therefore, partitioning the particles while 


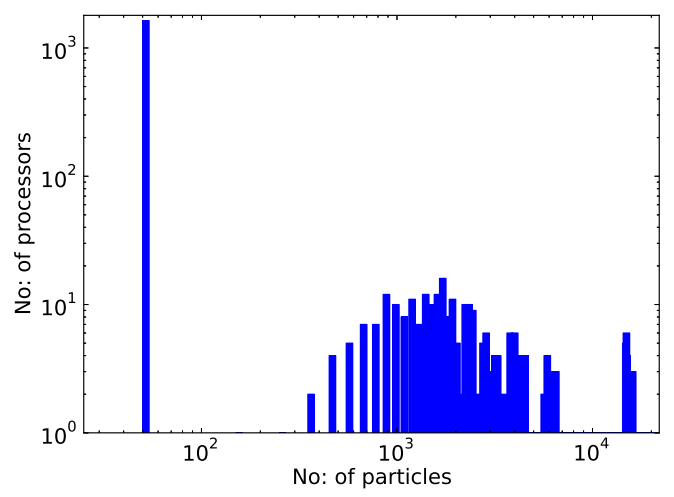

Figure 12: Rank distribution histogram with number of grid partition ranks that contain particle count between 0-100,100-200 and so on. These results are for a 1920 core run using the spatial decomposition algorithm.

preserving grid locality for a larger fraction becomes important. The performance of the locality optimization technique discussed in Section 4.1.3 is designed to take this into consideration. A comparison study regarding particle distribution and grid locality is done here for the spatial, particle sharing and equalization algorithms. The sensitivity of equalization algorithm parameters such as the number of iterations and coarse update step size to load balance and locality is also studied in this section. The runs in this section are done using 1920 ranks with seeding of 100,000 particles every 10 time steps. The simulation is run for a total of 200 time steps.

The rank distribution histogram for the spatial decomposition algorithm is shown in Fig. 12 where the number of grid partition ranks are counted for particle bin size equal to 100 . It is seen that more than 1600 ranks have a particle count between 0 and 100 while only a few grid partitions $\sim 10$ have large particle count between 14000-16000 particles.

Fig. 13 shows the comparison of average number of particles and the 

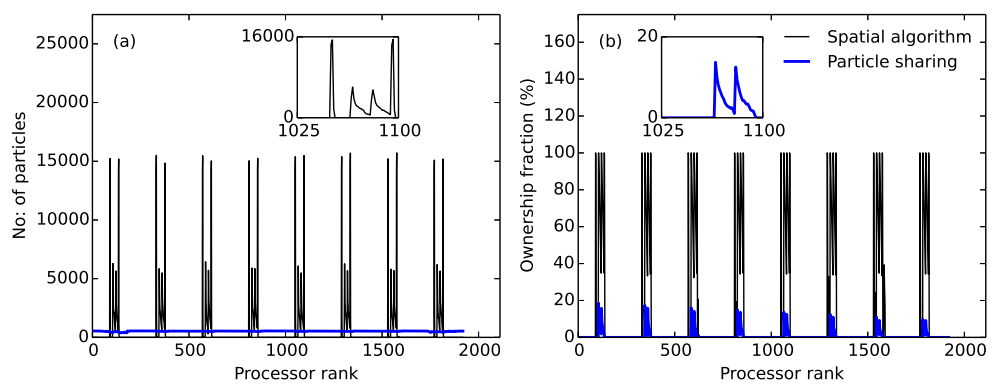

Figure 13: (a) average number of particles per MPI rank and (b) average fraction of particles that are local to its grid partition for a simulation using 1920 compute cores using spatial and particle sharing algorithm. The inset plot in (a) shows particle count data for the spatial algorithm and (b) shows the fraction of local particles for the particle sharing algorithm in a zoomed-in region for clarity.

Table 5: Parameters used for the equalization algorithm with variable names from algorithm 3

\begin{tabular}{|c|c|}
\hline Parameters & Value \\
\hline Total number of equalization iterations (niter) & 2000 \\
Coarse update step size (cstep) & 25 \\
Time step interval for equalization & 10 \\
\hline
\end{tabular}

fraction of particles local to grid partition for the spatial and particle sharing algorithm. Both of the quantities plotted here are averaged over the total number of time steps for every rank. The spatial algorithm evidently has imbalance in the number of particles but has close to $100 \%$ grid locality while the particle sharing algorithm has balance in the number of particles but grid locality is only $12 \%$, as shown in Fig. 13(b). The particles tend to be spatially located among ranks along the center line of the computational domain, which correspond to the peaks in particle count shown in Fig. 13(a), whereas it is more or less constant for the particle sharing algorithm.

The grouping of ranks for the equalization algorithm is done so that each group contains cores that reside on each node in the supercomputer thereby 

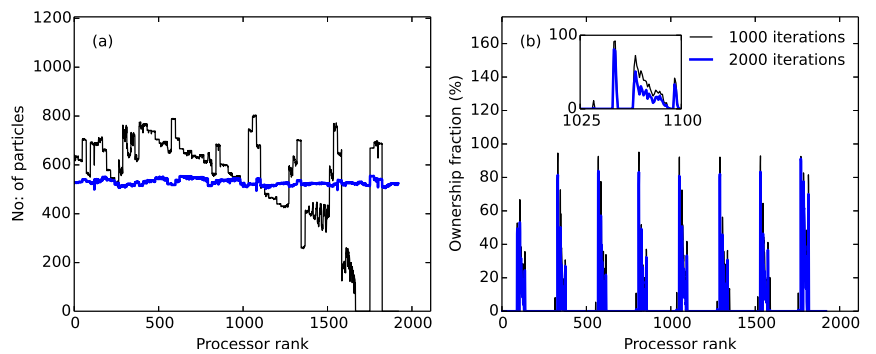

Figure 14: (a) average number of particles per MPI rank and (b) average fraction of particles that are local to its grid partition for a simulation using 1920 compute cores for particle sharing algorithm with 1000 and 2000 equalization iterations. The processor topology is $15 \times 16 \times 8$. The inset plot in (b) shows the same data in a zoomed-in region for clarity.

ensuring nodal locality. The number of cores per node on the Hopper is 24 which is used as the group size in these simulations. A coarse update iteration is interleaved between group equalization iterations to ensure balance of number of particles overall. The run time parameters for the equalization algorithm is shown in Table 5 .

The sensitivity of the equalization algorithm to the number of iterations (niter) is shown in Fig. 14. Larger number of iterations are required for effective balancing of particles as shown in Fig. 14(a). The fraction of particles local to the rank's grid partition is increased to $80-90 \%$ (Fig. 14(b)) for both the cases. This is significantly higher compared to grid locality fraction for the particle sharing algorithm shown in Fig. 13(b).

The sensitivity of the equalization method to the coarse update step size is shown in Fig. 15. The coarse update step size in Table 5 is varied while keeping other parameters constant. More frequent coarse updates resulted in better balance of particles among processors. Greater number of coarse updates also reduce the locality fraction because more number of particle are 

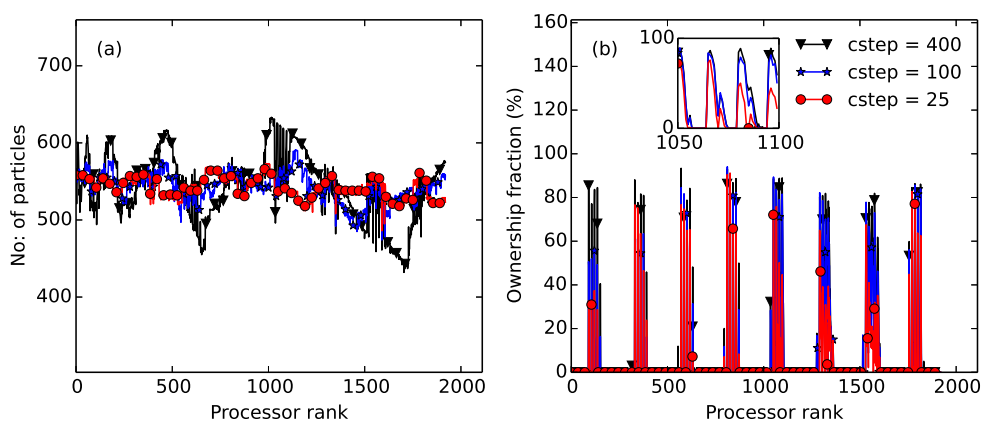

Figure 15: Sensitivity of (a) average number of particles per MPI rank and (b) average fraction of particles that are local to its grid partition to the coarse update step size (cstep). The inset plot in (b) shows the same data in a zoomed-in region for clarity.

Table 6: Equalization runtime parameters used for grid data movement study

\begin{tabular}{|c|c|}
\hline Parameters & Value \\
\hline Processor topology & $25 \times 16 \times 2$ \\
Total number of time steps & 300 \\
Time step interval for particle insertion & 10 \\
Number of particles inserted & 10000 \\
Time step interval for equalization & 50 \\
Total number of equalization iterations & 400 \\
Coarse update step size & 8 \\
\hline
\end{tabular}

exchanged among ranks.

The sensitivity of the equalization algorithm to time step interval over which it is applied is shown in Fig. 16. The time step interval in Table 5 is varied while keeping other parameters constant. Greater balance of particles and better data locality is attained when the equalization is applied more frequently during the simulation.

\subsubsection{Grid data movement}

The fact that improved data locality achieved by the equalization method, leads to less grid data transferred among ranks is studied here. The processor 

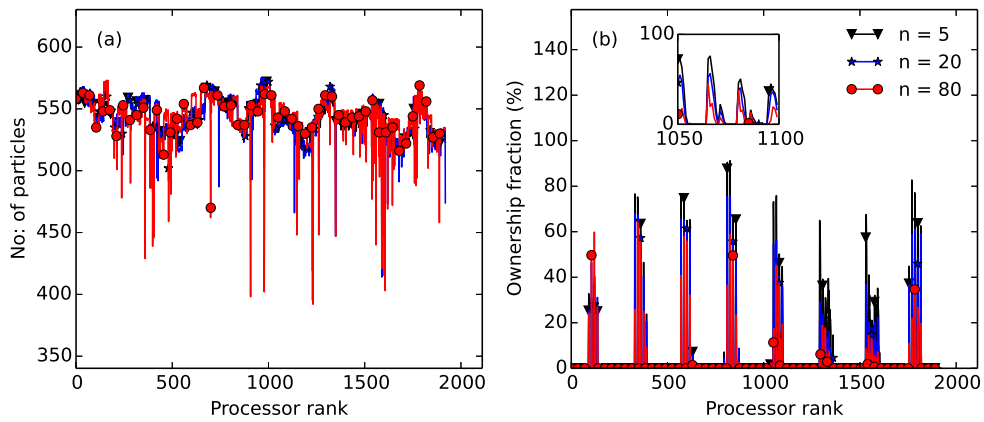

Figure 16: Sensitivity of (a) average number of particles per MPI rank and (b) average fraction of particles that are local to its grid partition to time step interval over which equalization is applied. The inset plots show the same data in a zoomed-in region for clarity.

topology, total number of time steps and the number of particles are chosen such that there is appreciable data transfer among processors. The parameters for this run are shown in Table 6 . The equalization algorithm preserves particle balance among ranks as shown in Fig. 17(a). It also tends to distribute particles in such a way that a larger fraction of particles that a rank owns is local to its grid partition. This redistribution of particles increases grid data locality and subsequently reduces the amount of data received per rank as shown Fig. 17(b). The equalization algorithm also results in efficient particle count balance for MPI ranks that lose particles through the computational domain boundaries. The reduction in the particle count seen in the "no equalization" case in Fig. 17(a) for ranks 150-250 and 550-650 are due to particle loss through the domain boundaries along the $\mathrm{z}$ direction. Fig. 17(c) compares the average grid data and average particle data received by each rank when the equalization algorithm is applied. The particle data transfer during equalization is four orders of magnitude less than grid data transfer. This is because the number of grid points vastly exceed the number 

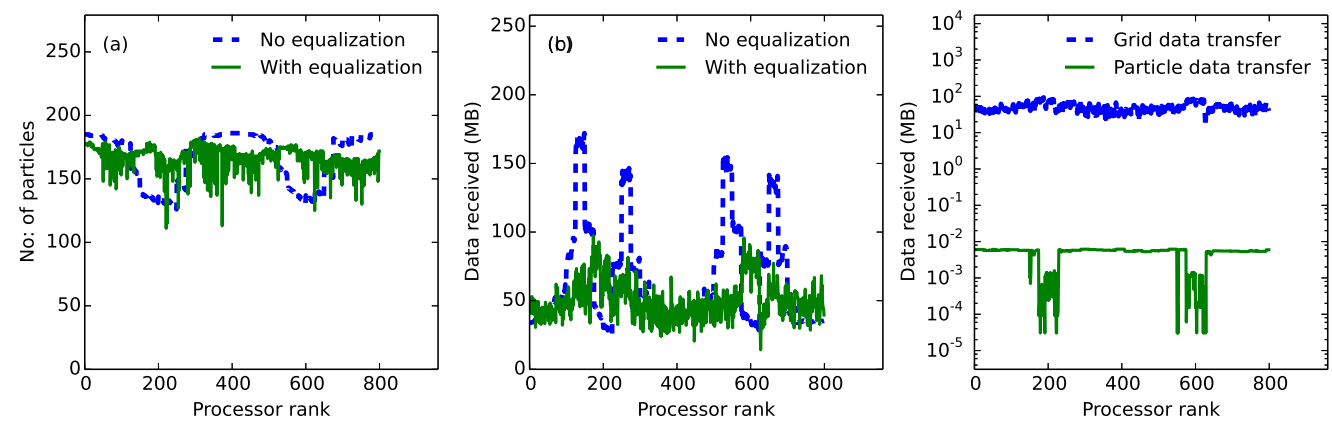

Figure 17: (a) number of particles per rank averaged over 300 time steps and (b) size in $\mathrm{MB}$ of grid data received per rank for the particle sharing algorithm with and without equalization.(c) Average grid and particle data in MB received by each rank during application of the equalization algorithm

Table 7: Run parameters used for Fig. 18. Particles are seeded in the domain every seed interval time steps.

\begin{tabular}{|c|c|c|c|c|c|}
\hline S/N & $\begin{array}{c}\text { time } \\
\text { steps }\end{array}$ & $\begin{array}{c}\text { communicator } \\
\text { size }\end{array}$ & $\begin{array}{c}\text { seed } \\
\text { interval }\end{array}$ & $\begin{array}{c}\text { Equalization } \\
\text { iterations (niter) }\end{array}$ & $\begin{array}{c}\text { Equalization } \\
\text { cost (ms) }\end{array}$ \\
\hline 1 & 200 & 6400 & 20 & 3200 & 93.4 \\
2 & 200 & 1600 & 20 & 800 & 18.4 \\
3 & 200 & 800 & 20 & 400 & 10.9 \\
4 & 300 & 800 & 10 & 400 & 15.1 \\
\hline
\end{tabular}

of particles. Particle data transfer is seen to drop among ranks $150-250$ and 550-650. These are the ranks which have particles local to its grid partition does not receive particles during equalization.

\subsubsection{Performance of equalization method}

The performance of the equalization algorithm is studied here with test cases selected in increasing order of data movement costs. These cases are shown in Table 7 . The number of particles seeded per seed interval is 10,000 and the equalization is done only once at half the total number of time steps. 


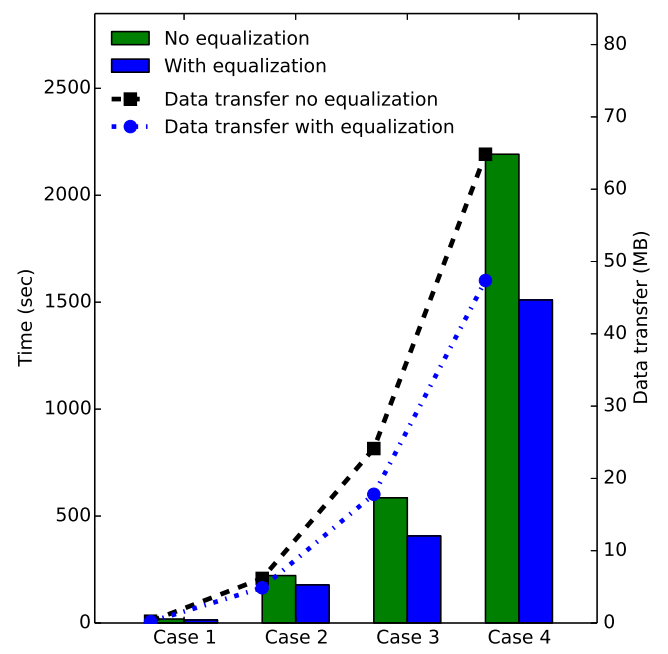

Figure 18: Simulation times for different runs with varying grid data transfer enlisted in Table 7 for the particle sharing algorithm with and without equalization. The data transfer in MB for the particle sharing algorithm with and without equalization is also plotted on secondary y axis on the right.

Fig. 18 compares the simulation times for varying average grid data transfer for four different runs. The parameters used for the runs are summarized in Table 7. The cost of equalization in milliseconds is also shown which is an extremely small fraction of total run time. The highest cost of equalization is approximately $0.6 \%$ of total simulation time for case 1 with 6400 ranks.

Grid data transfer is calculated as the total amount of grid data in MB, every rank sends, for a given time step. It is then averaged over all time steps and among all the ranks, as shown in Eq. 2. Here $d_{r i}$ represents the total data in MB sent by rank $r$ at time step $i . n$ and $p$ are the total number of time steps and ranks, respectively.

$$
D=\frac{1}{n p} \sum_{r=1}^{p} \sum_{i=1}^{n} d_{r i}
$$


It is seen that the grid locality significantly affects the simulation time for the particle sharing algorithm. Large grid data transfer implies lesser locality of particles to the grid partition owned by the rank. Particle sharing with equalization significantly reduces simulation time for the cases where there is large data transfer. A speed up close to a factor of 1.5 is seen for the case with the highest average grid data transfer of $64 \mathrm{MB}$. The grid data transfer with equalization is also lower compared to the runs without equalization. The particle sharing algorithm performs well without equalization when data transfer per rank is small, as shown at case 1 in Fig. 18. It is also seen that the equalization costs are higher for this case due to more equalization steps over larger number of processors.

\section{Comparison with existing implementations}

Zoltan [48] is a toolkit providing load balancing services with standard interfaces to a number of partitioning algorithms based on both coordinate- and graph/hyper graph-based approaches. A commonly used coordinate based option is the "Recursive Coordinate Bisection" (RCB) approach, where the workload is divided evenly by planes orthogonal to the coordinate directions. Applying this to a test dataset - a typical distribution of particles from the turbulent jet problem similar to Fig. 11(d) - produces a distribution with good spatial clustering of the particles assigned to each process, but no way to explicitly express the desire to have the particle clusters co-located with the necessary field data for the update. An alternative approach is to setup a graph partitioning problem where edges indicate an interaction between the objects. In this case, the algorithm attempts to balance the number of parti- 
cles per partition whilst simultaneously minimizing the number of edge cuts. The interface for Zoltan's native hyper graph partitioner allows us to assign specific particles to a fixed partition, so we can encode the locality requirement by adding edges between the particles and a 'pseudo-particle' object that is statically assigned to the specific rank representing the field data. Every edge cut then represents a non-local particle/field interaction. However, the desire for clustering/spatial locality is not explicitly enforced. The results of applying these three approaches (the equalization algorithm and the two Zoltan algorithms) are shown in Fig. 19 and 20. Twenty partitions with a processor topology of $5 \times 4 \times 1$ with approximately 20,000 particles is used in this test to make the results easier to interpret. Ranks 5 to 14 own grid partitions with co-located particles which can be seen from Fig. 19(a). In Fig. 19(b),(c) and (d), the initial particle distribution is shown on the outer circular ring, with the length of the segment indicating the number of particles assigned to that partition count, and is the same for all three cases. Similarly on the inner ring the length of the segments again indicates the particle count assigned to the labelled partition. The fractional distribution of particles before and after partitioning are shown as a line plot in Fig. 20 for the three different techniques. All of the algorithms produce a distribution with a similar number of particles per partition. The difference between the algorithms can be seen in the coloring of the bands mapping the particles from the initial to output distribution in Fig. 19. Bands where particles end up on the same partition as they started out (and hence are co-located with the field data necessary for the update) are shaded blue, whereas particles moving to different partition are shaded red. For this problem, the heuris- 


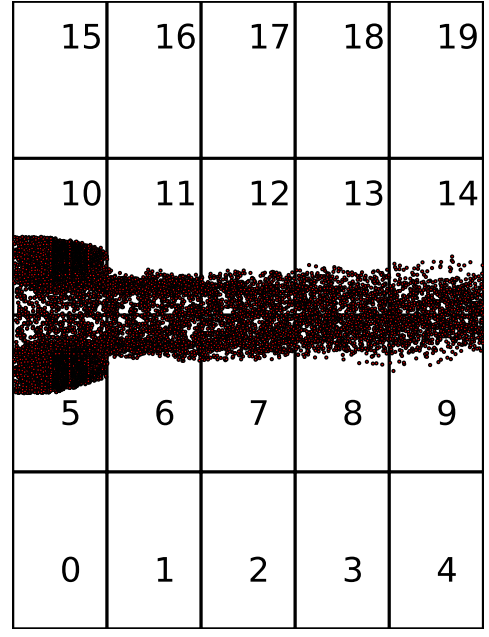

(a)

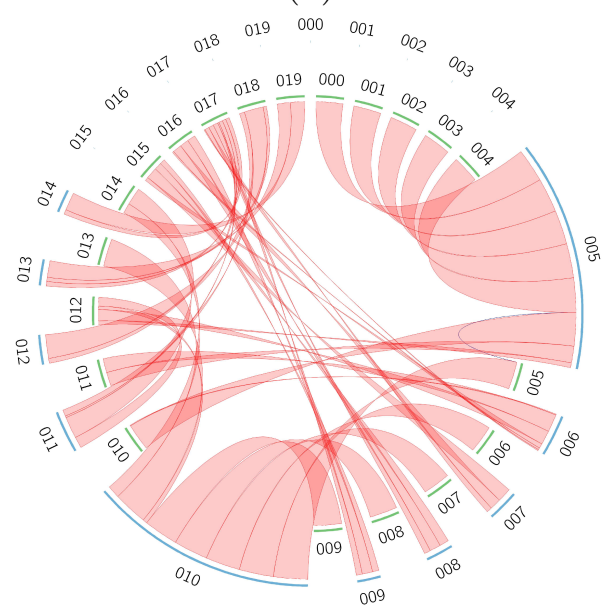

(c)

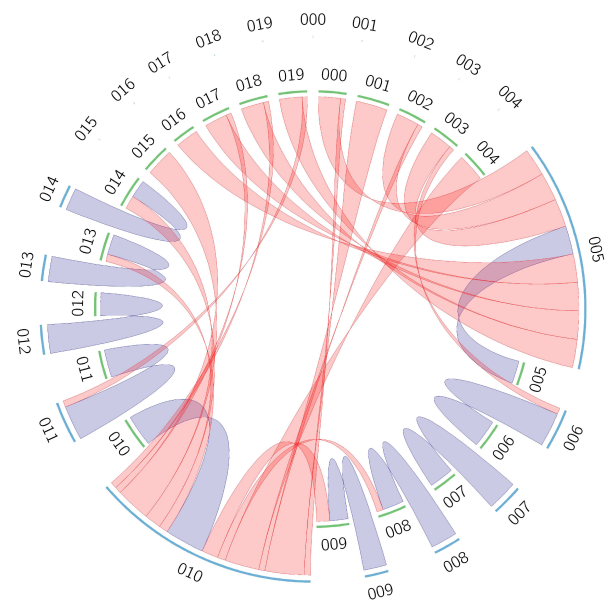

(b)

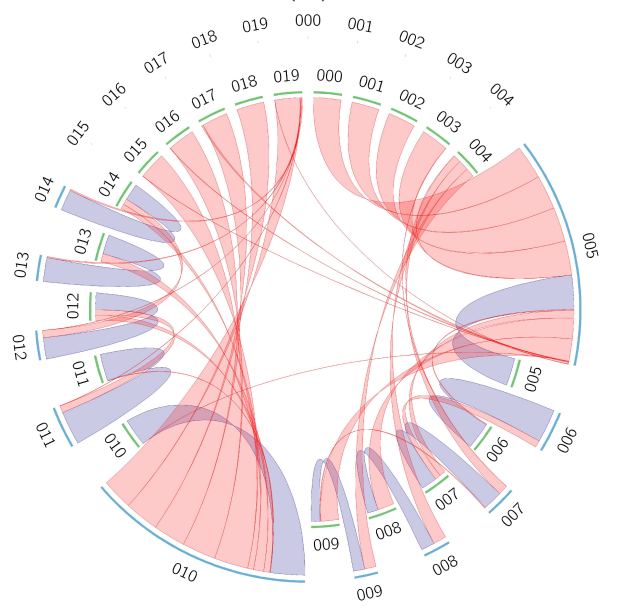

(d)

Figure 19: (a) Typical particle distribution and grid partitions for the turbulent jet problem using 20 ranks. Particle redistribution using (b) equalization (c) Zoltan (RCB) and (d) Zoltan (Graph) algorithms. Particles per process are shown on circular axis for the initial decomposition (outer ring, blue segments) where they are placed on the same process as the associated field data as well as the distribution after rebalancing (inner ring, green segments); the labels indicate the node owning those particles. The mapping between the two distributions is shown by the connecting ribbons, where a blue ribbon indicates particles that stay on the same node as the field data and a red ribbon indicates particles that are migrated to a different process. Plots produced with the aid of Circos [49] 


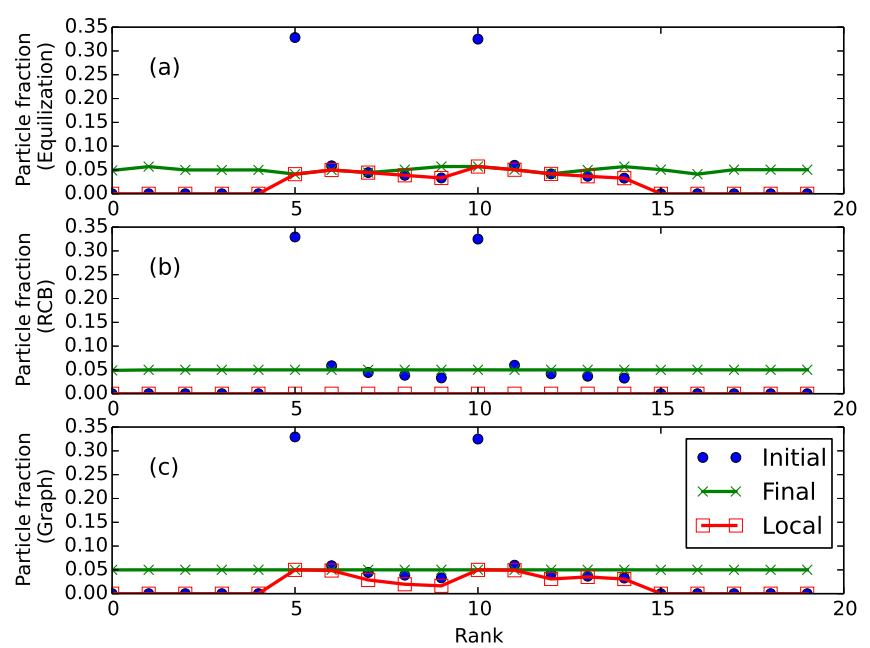

Figure 20: Fraction of particles before and after partitioning and locality fraction for (a) equalization (b) Zoltan (RCB) and (c) Zoltan (Graph) algorithms

tics embedded in the equalization approach are able to produce a favorable distribution relative to the more general Zoltan algorithms. While the RCB approach may have good spatial clustering, all of the particles end up nonlocal relative to their field data. The graph partitioning approach is much better than the RCB approach, however, there are several occurrences where by inspection we can see the solution is sub-optimal. For example, some particles are moved from partition 7,8 , and 9 to populate partition 4 , leaving these partitions short of the target particle count and requiring particles from partition 5. In contrast, the equalization strategy fills partition 4 from a single source (10) and keeps all of the particles local to ranks 7, 8, and 9 in place. The overall grid locality of particles is comparitively higher for the equalization algorithm, as shown in Fig. 20(a). The grid-locality fraction is virtually zero for the zoltan ( $\mathrm{RCB}$ ) algorithm, while the graph partitioning 
algorithm yields comparable locality fractions as the equalization method.

\section{Conclusions and future work}

Load balancing strategies for particle tracking algorithms on parallel distributed processors are studied in this paper. Particle tracking with two different velocity fields from turbulent fluid flow (homogeneous isotropic turbulence box and a turbulent jet) is used in one sided coupled particle simulations using two different algorithms. The performance of the spatial algorithm, where particles are co-located with the spatial grid decomposition, is compared with the particle sharing algorithm, where particles are shared equally among all MPI ranks irrespective of their spatial location. Sharing equal number of particles among all ranks showed improved parallel performance for the isotropic turbulence box problem after re-implementation of collective opertions, despite the loss of grid data locality. This problem is a case where particle count is significantly greater than number of grid points. The opposite situation where the number of grid points vastly exceed the number of particles is considered in the turbulent jet case where grid data transfer between MPI ranks becomes important. A hierarchical load balancing equalization algorithm to improve upon the particle sharing technique is formulated where particles are equally distributed among all MPI ranks while increasing grid locality. The use of equalization within the particle sharing method increases grid data locality as well as balances the number of particles among all MPI ranks. This leads to the reduction of interpolation grid data sent across ranks and significantly improves the performance of the particle sharing method. 
Future work will involve optimization of equalization algorithm parameters such as iteration count and coarse update step size to enhance performance. These experments were performed on a Gemini interconnect cluster and further validation studies may be required on other architectures such Blue Gene and Infiniband clusters. Further improvement in performance of MPI collectives can be achieved through UPC and Co-array fortran which will be explored in future studies.

\section{Acknowledgements}

This research used resources of the National Energy Research Scientific Computing Center, which is supported by the Office of Science of the U.S. Department of Energy under Contract No. DE-AC02-05CH11231. This research was supported by the Department of Energy Office of Advanced Scientific Computing Research.

[1] G. Gouesbet, A. Berlemont, Eulerian and Lagrangian approaches for predicting the behaviour of discrete particles in turbulent flows, Progress in Energy and Combustion Science 25 (2) (1998) 133-159.

[2] A. Berlemont, P. Desjonqueres, G. Gouesbet, Particle Lagrangian simulation in turbulent flows, International Journal of Multiphase Flow 16 (1) (1990) 19-34.

[3] V. Raman, H. Pitsch, R. Fox, Hybrid large-eddy simulation/Lagrangian filtered-density-function approach for simulating turbulent combustion, Combustion and Flame 143 (1) (2005) 56-78. 
[4] H. Yu, C. Wang, R. Grout, J. Chen, K. Ma, In situ visualization for large-scale combustion simulations, Computer Graphics and Applications, IEEE 30 (3) (2010) 45-57.

[5] P. Liewer, V. Decyk, A general concurrent algorithm for plasma particlein-cell simulation codes, Journal of Computational Physics 85 (2) (1989) $302-322$.

[6] E. Carmona, L. Chandler, On parallel PIC versatility and the structure of parallel PIC approaches, Concurrency: Practice and Experience 9 (12) (1997) 1377-1405.

[7] S. Plimpton, D. Seidel, M. Pasik, R. Coats, G. Montry, A load-balancing algorithm for a parallel electromagnetic particle-in-cell code, Computer Physics Communications 152 (3) (2003) 227-241.

[8] C. Nieter, J. Cary, Vorpal: A versatile plasma simulation code, Journal of Computational Physics 196 (2) (2004) 448-473.

[9] G. Bryan, M. Norman, A hybrid AMR application for cosmology and astrophysics, in: Structured Adaptive Mesh Refinement (SAMR) Grid Methods, Springer, 2000, pp. 165-170.

[10] A. Almgren, J. Bell, M. Lijewski, Z. Lukić, E. Van Andel, Nyx: A massively parallel AMR code for computational cosmology, The Astrophysical Journal 765 (1) (2013) 39.

[11] D. Pugmire, H. Childs, C. Garth, S. Ahern, G. Weber, Scalable computation of streamlines on very large datasets, in: High Performance 
Computing Networking, Storage and Analysis, Proceedings of the Conference on, IEEE, 2009, pp. 1-12.

[12] M. Boivin, O. Simonin, K. D. Squires, Direct numerical simulation of turbulence modulation by particles in isotropic turbulence, Journal of Fluid Mechanics 375 (1998) 235-263.

[13] N. A. Qazi, J. C. K. Tang, E. R. Hawkes, G. H. Yeoh, R. W. Grout, H. Sitaraman, M. Talei, R. A. Taylor, M. Bolla, H. Wang, Direct numerical simulation of hot solid particles in decaying isotropic turbulence., in: 19th Australasian Fluid Mechanics Conference (AFMC), Melbourne, Austrialia, 2014.

[14] S. Ethier, W. Tang, Z. Lin, Gyrokinetic particle-in-cell simulations of plasma microturbulence on advanced computing platforms, in: Journal of Physics: Conference Series, Vol. 16, IOP Publishing, 2005, p. 1.

[15] V. Springel, S. White, A. Jenkins, C. Frenk, N. Yoshida, L. Gao, J. Navarro, R. Thacker, D. Croton, J. Helly, et al., Simulations of the formation, evolution and clustering of galaxies and quasars, Nature 435 (7042) (2005) 629-636.

[16] X. Yuan, C. Salisbury, D. Balsara, R. Melhem, A load balancing package on distributed memory systems and its application to particle-particle particle-mesh (p3m) methods, Parallel Computing 23 (10) (1997) 15251544 .

[17] K. Bowers, R. Dror, D. Shaw, The midpoint method for parallelization 
of particle simulations, The Journal of Chemical Physics 124 (18) (2006) 184109.

[18] R. Fonseca, S. Martins, L. Silva, J. Tonge, F. Tsung, W. Mori, One-toone direct modeling of experiments and astrophysical scenarios: pushing the envelope on kinetic plasma simulations, Plasma Physics and Controlled Fusion 50 (12) (2008) 124034.

[19] J. Zhou, D. Liu, C. Liao, Z. Li, Chipic: An efficient code for electromagnetic pic modeling and simulation, Plasma Science, IEEE Transactions on 37 (10) (2009) 2002-2011.

[20] Q. Lu, D. Cai, Implementation of parallel plasma particle-in-cell codes on PC cluster, Computer Physics Communications 135 (1) (2001) 93104.

[21] J. Qiang, M. Furman, R. Ryne, A parallel particle-in-cell model for beam-beam interaction in high energy ring colliders, Journal of Computational Physics 198 (1) (2004) 278-294.

[22] A. Vishnu, M. ten Bruggencate, R. Olson, Evaluating the potential of cray gemini interconnect for pgas communication runtime systems, in: High Performance Interconnects (HOTI), 2011 IEEE 19th Annual Symposium on, IEEE, 2011, pp. 70-77.

[23] P. Narayanan, A. Koniges, L. Oliker, R. Preissl, S. Williams, N. Wright, B. Dudson, J. Candy, Performance characterization for fusion co-design applications, in: Proceedings of Cray Users Group, 2011. 
[24] P. Romano, B. Forget, F. Brown, Towards scalable parallelism in monte carlo particle transport codes using remote memory access, in: Joint International Conference on Supercomputing in Nuclear Applications and Monte Carlo, Vol. 2010, 2010, pp. 17-20.

[25] H. Shan, N. Wright, J. Shalf, K. Yelick, M. Wagner, N. Wichmann, A preliminary evaluation of the hardware acceleration of the Cray Gemini interconnect for PGAS languages and comparison with MPI, ACM SIGMETRICS Performance Evaluation Review 40 (2) (2012) 92-98.

[26] C. Othmer, J. Schüle, Dynamic load balancing of plasma particle-in-cell simulations: The taskfarm alternative, Computer Physics Communications 147 (1) (2002) 741-744.

[27] J. Singh, C. Holt, T. Totsuka, A. Gupta, J. Hennessy, Load balancing and data locality in adaptive hierarchical n-body methods: Barnes-hut, fast multipole, and radiosity, Journal of Parallel and Distributed Computing 27 (2) (1995) 118-141.

[28] J. Dubinski, J. Kim, C. Park, R. Humble, Gotpm: a parallel hybrid particle-mesh treecode, New Astronomy 9 (2) (2004) 111-126.

[29] C. Burstedde, O. Ghattas, M. Gurnis, T. Isaac, G. Stadler, T. Warburton, L. Wilcox, Extreme-scale amr, in: Proceedings of the 2010 ACM/IEEE International Conference for High Performance Computing, Networking, Storage and Analysis, IEEE Computer Society, 2010, pp. 1-12. 
[30] M. Berzins, J. Luitjens, Q. Meng, T. Harman, C. Wight, J. Peterson, Uintah: a scalable framework for hazard analysis, in: Proceedings of the 2010 TeraGrid Conference, ACM, 2010, p. 3.

[31] J. Pilkington, S. Baden, Dynamic partitioning of non-uniform structured workloads with spacefilling curves, Parallel and Distributed Systems, IEEE Transactions on 7 (3) (1996) 288-300.

[32] J. Chen, A. Choudhary, B. De Supinski, M. DeVries, E. Hawkes, S. Klasky, W. Liao, K. Ma, J. Mellor-Crummey, N. Podhorszki, et al., Terascale direct numerical simulations of turbulent combustion using S3D, Computational Science \& Discovery 2 (1) (2009) 015001.

[33] M. Willebeek-LeMair, A. Reeves, Strategies for dynamic load balancing on highly parallel computers, Parallel and Distributed Systems, IEEE Transactions on 4 (9) (1993) 979-993.

[34] J. Watts, S. Taylor, A practical approach to dynamic load balancing, Parallel and Distributed Systems, IEEE Transactions on 9 (3) (1998) $235-248$.

[35] P. Berenbrink, T. Friedetzky, Z. Hu, A new analytical method for parallel, diffusion-type load balancing, Journal of Parallel and Distributed Computing 69 (1) (2009) 54-61.

[36] J. Watts, S. Taylor, S. Nilpanich, Scplib: A concurrent programming library for programming heterogeneous networks of computers, in: Information Technology Conference, 1998. IEEE, IEEE, 1998, pp. 153-156. 
[37] National Energy Research Scientific Computing Center (NERSC), Office of Science and US Department of Energy, Contract No. DE-AC02$05 \mathrm{CH} 11231$.

[38] A. Bhagatwala, J. Chen, T. Lu, Direct numerical simulations of HCCI/SACI with ethanol, Combustion and Flame 161 (7) (2014) 18261841.

[39] R. Grout, A. Gruber, H. Kolla, P. Bremer, J. Bennett, A. Gyulassy, J. Chen, A direct numerical simulation study of turbulence and flame structure in transverse jets analysed in jet-trajectory based coordinates, Journal of Fluid Mechanics 706 (2012) 351-383.

[40] https://www.nersc.gov/users/computational-systems/nersc-8-systemcori/.

[41] Scientific grand challenges. architectures and technology for extreme scale computing, Tech. rep., Office of Advanced Scientific Computing, Office of Science and Office of Advanced Simulation and Computing, National Nuclear Security Administration, San Diego, CA (December 8-10 2009).

[42] J. Levesque, R. Sankaran, R. Grout, Hybridizing S3D into an exascale application using OpenACC: an approach for moving to multi-petaflops and beyond, in: Proceedings of the International conference on high performance computing, networking, storage and analysis, IEEE Computer Society Press, 2012, p. 15. 
[43] W. Gropp, E. Lusk, R. Thakur, Using MPI-2: Advanced features of the message-passing interface, Vol. 2, Globe Pequot, 1999.

[44] M. J. O'Brien, P. S. Brantley, K. I. Joy, Scalable load balancing for massively parallel distributed monte carlo particle transport, in: Proceedings of the 2013 International Conference on Mathematics and Computational Methods Applied to Nuclear Science and Engineering-M and C 2013, 2013.

[45] W. Gropp, Mpich2: A new start for mpi implementations, in: Recent Advances in Parallel Virtual Machine and Message Passing Interface, Springer, 2002, pp. 7-7.

[46] http://www.nersc.gov/users/computationalsystems/hopper/programming/compiling-codes-on-hopper/.

[47] C. Yoo, E. Richardson, R. Sankaran, J. Chen, A dns study on the stabilization mechanism of a turbulent lifted ethylene jet flame in highlyheated coflow, Proceedings of the Combustion Institute 33 (1) (2011) 1619-1627.

[48] E. G. Boman, U. V. Catalyurek, C. Chevalier, K. D. Devine, The Zoltan and Isorropia parallel toolkits for combinatorial scientific computing: Partitioning, ordering, and coloring, Scientific Programming 20 (2) (2012) 129-150.

[49] M. Krzywinski, J. Schein, I. Birol, J. Connors, R. Gascoyne, D. Horsman, S. Jones, M. Marra, Circos: an information aesthetic for comparative genomics, Genome Research 19 (9) (2009) 1639-1645. 